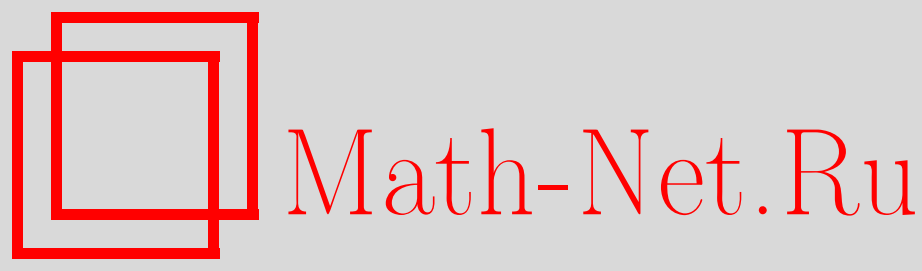

С. Л. Яковлев, Квантовая проблема $N$ тел: матричные структуры и уравнения, ТМФ, 2014, том 181, номер 1, 218-240

DOI: https://doi.org/10.4213/tmf8655

Использование Общероссийского математического портала Math-Net.Ru подразумевает, что вы прочитали и согласны с пользовательским соглашением http://www.mathnet.ru/rus/agreement

Параметры загрузки:

IP : 54.172 .240 .79

26 апреля 2023 г., 09:10:29

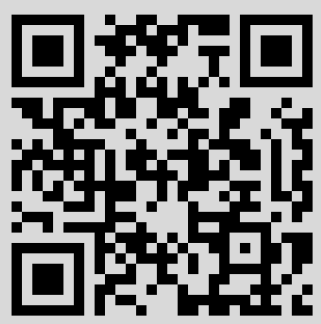




\title{
ФИЗИКА
}

Том 181, № 1

октябрь, 2014

(C) 2014 г.

\author{
С. Л. Яковлев*
}

\section{КВАНТОВАЯ ПРОБЛЕМА $N$ ТЕЛ: МАТРИЧНЫЕ СТРУКТУРЫ И УРАВНЕНИЯ}

\begin{abstract}
Рассматриваются матричные структуры в квантовой задаче $N$ тел, обобщающие компоненты Фаддеева для резольвент, $T$-матриц и собственных функций непрерывного спектра. Для введенных компонент $T$-матриц и резольвент выписаны матричные уравнения, и из этих уравнений получены матричные операторы, обобщающие на случай произвольного числа частиц матричные трехчастичные операторы Фаддеева. Определены собственные функции непрерывного спектра этих матричных операторов.
\end{abstract}

Ключевые слова: квантовая проблема $N$ тел, интегральные уравнения Фаддеева, интегральные и дифференциальные уравнения для компонент волновых функций, резольвент, $T$-матриц.

DOI: $10.4213 / \operatorname{tmf} 8655$

Посвящается восъмидесятилетнему юбилею академика Л. Д. Фаддеева

\section{1. ВВЕДЕНИЕ И ПОСТАНОВКА ЗАДАЧИ}

В настоящей работе проведено систематическое обобщение формализма матричных уравнений Фаддеева для трех тел на случай произвольного числа частиц. Первоначально уравнения Фаддеева появились в форме интегральных уравнений для $(3 \times 3)$-матричных ядер, отвечающих компонентам $T$-матрицы [1], [2]. Затем в ряде работ [3]-[9] были предприняты попытки обобщить эти уравнения на системы из большего числа частиц. И только в работах [10]-[12] такое обобщение было построено. Впоследствии в работах [13]-[15] было найдено обобщение уравнений Фаддеева на случай $N$ частиц также в дифференциальной форме, а в работах [16]-[19] были развиты методы решения этих уравнений для конкретных систем.

Следует отметить, что необходимость построения матричных уравнений со специальной структурой зацепления уравнений вытекает из хорошо известного факта, что интегральное уравнение Липпманна-Швингера начиная со случая трех частиц теряет свойство фредгольмовости. А поскольку это уравнение является основным средством для построения решений стационарной задачи рассеяния в случае двух частиц, требовалось найти ему замену. Трехчастичные интегральные уравнения

${ }^{*}$ Санкт-Петербургский государственный университет, Санкт-Петербург, Россия. E-mail: sl-yakovlev@yandex.ru 
Фаддеева и стали искомой заменой, так как именно для этих уравнений свойство фредгольмовости было восстановлено. С матричными интегральными уравнениями Фаддеева естественным образом связаны матричные дифференциальные уравнения, подобно тому как уравнение Липпманна-Швингера связано с уравнением Шредингера. В случае трех частиц эта связь была прослежена в работах [20]-[22], где были построены матричные операторы, собственные функции которых дают компоненты Фаддеева волновых функций системы трех тел. При этом компоненты Фаддеева резольвент и $T$-матриц оказываются матричными элементами резольвент и $T$-матриц для этих операторов. Дальнейшее развитие данных результатов в случае произвольного числа частиц является предметом настоящей работы.

(. Оператор энергии системы $\$ N \$$ частиц, цепочки разбиений, относительные координаты)Оператор энергии системы $N$ частиц, цепочки разбиений, относительные координаты

Рассмотрим систему $N$ попарно взаимодействующих частиц. Оператор энергии имеет вид ${ }^{1)}$

$$
\widehat{H}=-\sum_{i=1}^{N} \frac{1}{2 m_{i}} \Delta_{\mathbf{r}_{i}}+\sum_{1 \leqslant i<j \leqslant N} v_{i j}\left(\mathbf{r}_{i}-\mathbf{r}_{j}\right),
$$

где $m_{i}$ и $\mathbf{r}_{i} \in \mathbb{R}^{3}$ - масса и радиус-вектор $i$-й частицы, $\Delta_{\mathbf{r}_{i}}$ - оператор Лапласа, функции $v_{i j}$ - потенциалы взаимодействия между частицами $i$ и $j$. В силу трансляционной инвариантности потенциальной энергии оператор $\widehat{H}$ содержит тривиальное слагаемое, которое отвечает свободному движению системы как целого. Стандартным способом явного отделения этого слагаемого является переход от координат частиц $\mathbf{r}_{1}, \ldots, \mathbf{r}_{N}$ к радиус-вектору центра масс

$$
\mathbf{R}=\frac{1}{M} \sum_{i=1}^{N} m_{i} \mathbf{r}_{i}, \quad M=\sum_{i=1}^{N} m_{i},
$$

и системе трансляционно-инвариантных относительных координат. Для описания последних, а также для классификации объектов, встречающихся в дальнейшем, необходимо ввести понятие цепочек разбиений.

Разбиением $a_{k}$ называется некоторый способ разделения системы $N$ частиц на $k$ подсистем:

$$
a_{k}=\left\{\omega_{1}, \ldots, \omega_{k}\right\}, \quad \omega_{i} \cap \omega_{j}=\varnothing, \quad \omega_{i} \subset\{1, \ldots, N\} .
$$

Порядок следования частиц в каждой из подсистем несуществен. Заметим, что существуют два тривиальных разбиения $a_{1}=\{1, \ldots, N\}$ и $a_{N}=\{(1), \ldots,(N)\}$, и тем самым нетривиальными являются все разбиения $a_{k}$ при $2 \leqslant k \leqslant N-1$. Соотношение $a_{k} \subset a_{l}$ при $k \geqslant l$ означает, что $a_{k}=a_{l}$ при $k=l$. Если $k=l+1$, то для разбиений $a_{l+1}=\left\{\omega_{1}, \ldots, \omega_{p}, \ldots, \omega_{q}, \ldots, \omega_{l+1}\right\}$ и $a_{l}=\left\{\sigma_{1}, \ldots, \sigma_{r}, \ldots, \sigma_{l}\right\}$ существуют такие номера $p, q$ и $r$, что $\omega_{p} \cup \omega_{q}=\sigma_{r}$. При $k>l+1$ существует последовательность разбиений $a_{k-1} \subset \cdots \subset a_{l+2} \subset a_{l+1}$ такая, что $a_{k} \subset a_{k-1}$ и $a_{l+1} \subset a_{l}$. В дальнейшем одинаковыми буквами обозначаются разбиения, связанные знаком $\subset$.

Цепочкой разбиений $A_{l}^{k}$ называется последовательность $A_{l}^{k}=a_{l}, a_{l+1}, \ldots, a_{k}$, которая составлена из нетривиальных разбиений с $2 \leqslant l<k \leqslant N-1$. В частных случаях $k=N-1$ и $l=2$ применяются обозначения $A_{l}=a_{l}, a_{l+1}, \ldots, a_{N-1}$

1) На протяжении всего текста используется система единиц, в которой постоянная Планка $\hbar=1$. 
и $A^{k}=a_{2}, a_{3}, \ldots, a_{k}$. Понятно, что самая полная информация о разбиениях системы $N$ частиц содержится в цепочках $A_{2}$.

Обозначим через $n_{N}$ общее число цепочек $A_{2}$ для системы $N$ частиц. Тогда легко сосчитать, что $n_{N}=2^{1-N} N !(N-1)$, в частности $n_{3}=3, n_{4}=18, n_{5}=180, n_{6}=2700$ и т. д.

Введем относительные координаты, которые обычно связывают с именем Якоби. Следует отметить, что для $N$ частиц существуют несколько различных типов относительных координат. При этом внутри каждого типа существуют несколько систем относительных координат. В настоящей работе выбран один из таких типов, в котором каждая из систем координат единственным образом фиксируется выбором цепочки разбиений. Таким образом мы определяем $n_{N}$ координатных систем в конфигурационном пространстве $\mathbb{R}^{d}, d=3 N-1$.

Пусть $A_{2}$ - некоторая цепочка разбиений. Определим $N-1$ векторов

$$
\mathbf{x}_{a_{2}}^{a_{1}}, \mathbf{x}_{a_{3}}^{a_{2}}, \ldots, \mathbf{x}_{a_{N}}^{a_{N-1}}
$$

по формуле

где

$$
\mathbf{x}_{a_{l}}^{a_{l-1}}=\left(\frac{2 M_{\omega_{p}} M_{\omega_{q}}}{M_{\omega_{p}}+M_{\omega_{q}}}\right)^{1 / 2}\left(\mathbf{R}_{\omega_{p}}-\mathbf{R}_{\omega_{q}}\right)
$$

$$
M_{\omega_{p}}=\sum_{j \in \omega_{p}} m_{j}, \quad \mathbf{R}_{\omega_{p}}=\frac{1}{M_{\omega_{p}}} \sum_{j \in \omega_{p}} m_{j} \mathbf{r}_{j}
$$

а подсистемы $\omega_{p}$ и $\omega_{q}$ определяются парой разбиений $a_{l-1}=\left\{\sigma_{1}, \ldots, \sigma_{r}, \ldots, \sigma_{l-1}\right\}$ и $a_{l}=\left\{\omega_{1}, \ldots, \omega_{p}, \ldots, \omega_{q}, \ldots, \omega_{l}\right\}$ так, что имеет место равенство $\omega_{p} \cup \omega_{q}=\sigma_{r}$.

Набор векторов $\mathbf{X}_{A_{2}}=\left\{\mathbf{x}_{a_{2}}^{a_{1}}, \ldots, \mathbf{x}_{a_{N}}^{a_{N-1}}\right\}$ совместно с вектором $\mathbf{R}$ из (1) дает альтернативные к набору $\left\{\mathbf{r}_{1}, \ldots, \mathbf{r}_{N}\right\}$ координаты для системы $N$ частиц. Заметим, что формулы (1) и (2) фактически определяют линейное преобразование

$$
\left\{\mathbf{R}, \mathbf{X}_{A_{2}}\right\}=L_{A_{2}}\left\{\mathbf{r}_{1}, \ldots, \mathbf{r}_{N}\right\}
$$

с невырожденной матрицей $L_{A_{2}}$ размера $N \times N$. Аналогично для некоторой цепочки $B_{2} \neq A_{2}$ имеем

$$
\left\{\mathbf{R}, \mathbf{X}_{B_{2}}\right\}=L_{B_{2}}\left\{\mathbf{r}_{1}, \ldots, \mathbf{r}_{N}\right\}
$$

Отсюда следует, что

$$
\left\{\mathbf{R}, \mathbf{X}_{B_{2}}\right\}=L_{B_{2}} L_{A_{2}}^{-1}\left\{\mathbf{R}, \mathbf{X}_{A_{2}}\right\} .
$$

Последнее означает, что матрица $L_{B_{2}} L_{A_{2}}^{-1}$ имеет блочную структуру $\operatorname{diag}\left[1, O_{B_{2} A_{2}}\right]$ с матрицей $O_{B_{2} A_{2}}$ размера $(N-1) \times(N-1)$, которая реализует преобразование относительных координат $\mathbf{X}_{A_{2}}$ и $\mathbf{X}_{B_{2}}$ :

$$
\mathbf{X}_{B_{2}}=O_{B_{2} A_{2}} \mathbf{X}_{A_{2}} .
$$

Матрица преобразования $O_{B_{2} A_{2}}$ является ортогональной, $O_{B_{2} A_{2}} O_{B_{2} A_{2}}^{\mathrm{T}}=I$, где Т означает транспонирование. Для доказательства достаточно заметить, что для любой цепочки $C_{2}$ выполняется равенство

$$
\sum_{i=1}^{N} m_{i} \mathbf{r}_{i}^{2}=M \mathbf{R}^{2}+\frac{1}{2} \sum_{j=2}^{N}\left(\mathbf{x}_{c_{j}}^{c_{j-1}}\right)^{2},
$$


откуда для любых $A_{2} \neq B_{2}$ следует, что

$$
\sum_{j=2}^{N}\left(\mathbf{x}_{a_{j}}^{a_{j-1}}\right)^{2}=\sum_{j=2}^{N}\left(\mathbf{x}_{b_{j}}^{b_{j-1}}\right)^{2} .
$$

Введем несколько более компактных обозначений для наборов относительных координат. Пусть $a_{k}-$ некоторое разбиение из цепочки $A_{2}$. Обозначим через $\mathbf{x}_{a_{k}}$ набор относительных координат, внутренних по отношению к подсистемам разбиения $a_{k}$ :

$$
\mathbf{x}_{a_{k}}=\left\{\mathbf{x}_{a_{k+1}}^{a_{k}}, \ldots, \mathbf{x}_{a_{N}}^{a_{N-1}}\right\}
$$

Через $\mathbf{y}_{a_{k}}$ обозначим набор внешних по отношению к $a_{k}$ относительных координат:

$$
\mathbf{y}_{a_{k}}=\left\{\mathbf{x}_{a_{2}}^{a_{1}}, \ldots, \mathbf{x}_{a_{k}}^{a_{k-1}}\right\} .
$$

По построению $\mathbf{X}_{A_{2}}=\left\{\mathbf{x}_{a_{k}}, \mathbf{y}_{a_{k}}\right\}$. Согласно определению $\mathbf{x}_{a_{N-1}}=\mathbf{x}_{a_{N}-1}^{a_{N-1}}$ и $\mathbf{y}_{a_{2}}=\mathbf{x}_{a_{2}}^{a_{1}}$. Наконец, заметим, что

$$
\mathbf{x}_{a_{N-1}}=\left(\frac{2 m_{i} m_{j}}{m_{i}+m_{j}}\right)^{1 / 2}\left(\mathbf{r}_{i}-\mathbf{r}_{j}\right),
$$

где $i$ и $j$ - номера частиц, входящих в единственную нетривиальную подсистему разбиения $a_{N-1}$. В дальнейшем мы будем систематически использовать взаимно однозначное соответствие между парами $i j$ и разбиениями $a_{N-1}$ для классификации различных парных объектов.

Введенные координаты позволяют переписать оператор $\widehat{H}$ в виде

$$
\widehat{H}=-\frac{1}{2 M} \Delta_{\mathbf{R}}-\sum_{k=2}^{N} \Delta_{\mathbf{x}_{a_{k}}^{a_{k-1}}}+\sum_{b_{N-1}} V_{b_{N-1}}\left(\mathbf{x}_{b_{N-1}}\right),
$$

где $V_{b_{N-1}}\left(\mathbf{x}_{b_{N-1}}\right)=v_{i j}\left(\mathbf{r}_{i}-\mathbf{r}_{j}\right)$, а $i, j-$ номера частиц, входящих в единственную нетривиальную подсистему разбиения $b_{N-1}$. Здесь и в дальнейшем предполагается, что фиксирована некоторая цепочка разбиений $A_{2}$, которая определяет набор координат $\mathbf{X}_{A_{2}}$, а векторы $\mathbf{x}_{b_{N-1}}$ в последней сумме выражены в терминах координат $\mathbf{X}_{A_{2}}$ по формулам (3). Сумму трехмерных операторов Лапласа $\sum_{k=2}^{N} \Delta_{\mathbf{x}_{a_{k}}}{ }_{k-1}$ удобно объединить в лапласиан $\Delta_{\mathbf{X}_{A_{2}}}$, где $\mathbf{X}_{A_{2}} \in \mathbb{R}^{d}$. Благодаря ортогональности преобразования (3) для любых $A_{2} \neq B_{2}$ выполняется равенство

$$
\Delta_{\mathbf{X}_{A_{2}}}=\Delta_{\mathbf{X}_{B_{2}}} .
$$

По этой причине в дальнейшем мы будем опускать индексы цепочек у координат $\mathbf{X}_{A_{2}}$ и писать просто $\mathbf{X}$. Заметим, что для любого $a_{k}$ верно представление

$$
\Delta_{\mathbf{X}}=\Delta_{\mathbf{x}_{a_{k}}}+\Delta_{\mathbf{y}_{a_{k}}},
$$

где $\mathbf{x}_{a_{k}} \in \mathbb{R}^{3(N-k)}$ и $\mathbf{y}_{a_{k}} \in \mathbb{R}^{3(k-1)}$ - внутренние и внешние по отношению к $a_{k}$ координаты, определенные выше.

Отделяя в (4) слагаемое $-(2 M)^{-1} \Delta_{\mathbf{R}}$, отвечающее свободному движению системы $N$ частиц как целого, получаем оператор энергии $N$ частиц в системе центра масс:

$$
H=-\Delta_{\mathbf{X}}+\sum_{b_{N-1}} V_{b_{N-1}}\left(\mathbf{x}_{b_{N-1}}\right) \equiv H_{0}+\sum_{b_{N-1}} V_{b_{N-1}} .
$$


Именно оператор $H$ определяет все физические свойства системы, поэтому его изучение и представляет основную задачу.

Оператор $H$ рассматривается в гильбертовом пространстве $\mathcal{H}=L_{2}\left(\mathbb{R}^{d}\right)$ квадратично-интегрируемых функций, зависящих от относительных координат. Предполагается, что потенциалы взаимодействия $V_{b_{N-1}}\left(\mathbf{x}_{b_{N-1}}\right)$ являются вещественнозначными, гладкими, достаточно быстро убывающими функциями при $\left|\mathbf{x}_{b_{N-1}}\right| \rightarrow \infty$ :

$$
\left|V_{b_{N-1}}(\mathbf{x})\right| \leqslant C(1+|\mathbf{x}|)^{-(3+\mu)}, \quad \mu>0 .
$$

Оператор $H$ с такими потенциалами становится самосопряженным в пространстве $\mathcal{H}$ с областью определения $D=D\left(-\Delta_{\mathbf{X}}\right)$ [1], [23]. Потенциалы, удовлетворяющие условиям (7), не включают кулоновский случай, который требует специальных модификаций формализма уравнений Фаддеева [2], [24], что выходит за рамки настоящей работы.

Наряду с оператором $H$ будем рассматривать операторы вида

$$
H_{a_{k}}=H_{0}+\sum_{a_{N-1} \subset a_{k}} V_{a_{N-1}} .
$$

Эти операторы соответствуют системам $N$ частиц, в которых взаимодействуют между собой лишь частицы, принадлежащие одной и той же подсистеме разбиения $a_{k}$, тогда как взаимодействие между частицами из разных подсистем отсутствует. Для операторов $H_{a_{k}}$ в силу равенства (5) имеет место представление

$$
H_{a_{k}}=h_{a_{k}}-\Delta_{\mathbf{y}_{a_{k}}}, \quad h_{a_{k}}=-\Delta_{\mathbf{x}_{a_{k}}}+\sum_{a_{N-1} \subset a_{k}} V_{a_{N-1}}\left(\mathbf{x}_{a_{N-1}}\right) .
$$

Итак, мы описали операторы $H$ и $H_{a_{k}}$ в координатном пространстве. Переход в импульсное представление осуществляется с помощью преобразования Фурье (чтобы избежать недоразумений, для мнимой единицы далее используется обозначение і)

$$
f(\mathbf{X})=\frac{1}{\sqrt{(2 \pi)^{d}}} \int d \mathbf{P} e^{\mathrm{i}(\mathbf{P}, \mathbf{X})} f(\mathbf{P}),
$$

где $\mathbf{P} \in \mathbb{R}^{d}$ - набор относительных импульсов $\mathbf{k}_{a_{2}}^{a_{1}}, \ldots, \mathbf{k}_{a_{N}}^{a_{N-1}}$, сопряженных координатам $\mathbf{X}$. Оператор $H$ в импульсном пространстве дается выражением (6), где

$$
H_{0}=\mathbf{P}^{2}=\mathbf{k}_{a_{k}}^{2}+\mathbf{p}_{a_{k}}^{2}
$$

a $V_{a_{N-1}}-$ интегральные операторы с ядрами

$$
V_{a_{N-1}}\left(\mathbf{P}, \mathbf{P}^{\prime}\right)=V_{a_{N-1}}\left(\mathbf{k}_{a_{N-1}}-\mathbf{k}_{a_{N-1}}^{\prime}\right) \delta\left(\mathbf{p}_{a_{N-1}}-\mathbf{p}_{a_{N-1}}^{\prime}\right) .
$$

Функции $V_{a_{N-1}}(\mathbf{k})$ связаны с $V_{a_{N-1}}(\mathbf{x})$ преобразованием Фурье:

$$
V_{a_{N-1}}(\mathbf{k})=\frac{1}{(2 \pi)^{3}} \int d \mathbf{x} e^{\mathrm{i}(\mathbf{k}, \mathbf{x})} V_{a_{N-1}}(\mathbf{x}), \quad \mathbf{k}, \mathbf{x} \in \mathbb{R}^{3} .
$$

Для операторов $H, H_{a_{k}}$ и $H_{0}$ резольвенты определяются равенствами

$$
R(z)=(H-z I)^{-1}, \quad R_{a_{k}}(z)=\left(H_{a_{k}}-z I\right)^{-1}, \quad R_{0}=\left(H_{0}-z I\right)^{-1},
$$


где $I$ - единичный оператор в пространстве $\mathcal{H}$. Отвечающие резольвентам $T$-матрицы имеют вид

$$
T(z)=V-V R(z) V, \quad T_{a_{k}}=V_{a_{k}}-V_{a_{k}} R_{a_{k}}(z) V_{a_{k}},
$$

где

$$
V=\sum_{a_{N-1}} V_{a_{N-1}}, \quad V_{a_{k}}=\sum_{a_{N-1} \subset a_{k}} V_{a_{N-1}} .
$$

Обратное преобразование

$$
R(z)=R_{0}(z)-R_{0}(z) T(z) R_{0}(z), \quad R_{a_{k}}(z)=R_{0}(z)-R_{0}(z) T_{a_{k}}(z) R_{0}(z)
$$

получается стандартными методами.

Как обычно, при решении задачи $N$ тел предполагаются известными решения задач для меньшего числа частиц, в частности, при изучении резольвенты $R(z)$ считаются известными все операторы $R_{a_{k}}(z)$ при $2 \leqslant k \leqslant N-1$. Однако, как и в работе [10], мы выписываем соответствующие уравнения для всех операторов $R_{a_{l}}(z)$. При этом самые сложные уравнения для $R(z)$ получаются при $l=1$.

В конце данного раздела условимся о некоторых обозначениях, используемых на протяжении работы. Как ясно из предыдущего, векторы будем обозначать жирными буквами, их модули как $|\mathbf{x}|$, а единичные векторы - как $\hat{\mathbf{x}}=\mathbf{x} /|\mathbf{x}|$. Скалярные произведения векторов обозначаются через $(\mathbf{x}, \mathbf{y})$.

\section{2. КОМПОНЕНТЫ Т-МАТРИЦЫ И РЕЗОЛЬВЕНТЫ И УРАВНЕНИЯ ФАДДЕЕВА-ЯКУБОВСКОГО}

В настоящем разделе определяются компоненты $T$-матриц $T(z), T_{a_{k}}(z)$ и резольвент $R(z), R_{a_{k}}(z)$. Компоненты классифицируются цепочками разбиений $A_{l}$. Такие компоненты и уравнения для них были введены в работе [1] для $N=3$ и затем обобщены на случай произвольных $N$ в статьях [10], [11]. Будем работать с операторами $T_{a_{k}}(z)$ и $R_{a_{k}}(z)$, проведя отождествления $T_{a_{1}}(z)=T(z)$ и $R_{a_{1}}(z)=R(z)$.

Компоненты $T$-матриц $T_{a_{k}}$ определяются следующими рекуррентными соотношениями:

$$
\begin{aligned}
M_{a_{N-1} b_{N-1}}^{a_{k}}(z)= & V_{a_{N-1} \delta_{a_{N-1} b_{N-1}}-V_{a_{N-1}} R_{a_{k}}(z) V_{b_{N-1}},} \\
M_{A_{i-1} B_{i-1}}^{a_{k}}(z)= & M_{A_{i+1} B_{i+1}}^{a_{i}}(z) \delta_{a_{i} b_{i}} \delta_{a_{i-1} b_{i-1}}- \\
& -\sum_{\left(d_{i} \neq a_{i}\right) \subset a_{i-1}} \sum_{\left(C_{i+1} \neq D_{i+1}\right) \subset a_{i}}^{\prime} M_{A_{i+1} C_{i+1}}^{a_{i}}(z) R_{0}(z) M_{D_{i} B_{i}}^{a_{k}}(z),
\end{aligned}
$$

где $A_{i} \subset a_{k}, B_{i} \subset a_{k}, k+1 \leqslant i \leqslant N-1,1 \leqslant k \leqslant N-1$ и $\delta_{a_{i} b_{i}}$ - символ Кронекера. Штрих у знака суммы означает, что ограничения при суммировании понимаются покомпонентно: цепочки $C_{i+1}$ и $D_{i+1}$ подчиняются условиям

$$
c_{N-1} \neq d_{N-1}, \ldots, c_{i+1} \neq d_{i+1}, \quad d_{N-1} \subset c_{N-2}, \ldots, d_{i+2} \subset c_{i+1} .
$$

Операторы $M_{A_{k} B_{k}}^{a_{i}}(z)$ удовлетворяют системе уравнений [10], [11]

$$
\begin{aligned}
M_{A_{k} B_{k}}^{a_{i}}(z)= & M_{A_{k+1} B_{k+1}}^{a_{k}}(z) \delta_{a_{k} b_{k}}- \\
& -\sum_{d_{k} \neq a_{k}\left(C_{k+1} \neq D_{k+1}\right) \subset a_{k}}^{\prime} \sum_{A_{k+1} C_{k+1}}^{a_{k}}(z) R_{0}(z) M_{D_{k} B_{k}}^{a_{i}}(z) .
\end{aligned}
$$


В случае $k=2, i=1$ уравнения (9) являются обобщением системы уравнений Фаддеева [1] на случай $N$ частиц. Сформулируем два важнейших свойства уравнений (9), доказанных в работах [10], [11].

Лемма 1. Пусть операторь $\widetilde{M}_{A_{k} B_{k}}^{a_{i}}(z), \operatorname{Im} z \neq 0$, определены на $D$ и удовлетворяют системе (9). Тогда операторы

$$
\widetilde{M}_{A_{k+1} B_{k+1}}^{a_{i}}(z)=\sum_{a_{k}} \widetilde{M}_{A_{k} B_{k}}^{a_{i}}(z), \quad \widetilde{T}_{a_{i}}(z)=\sum_{A_{k}, b_{N-1}} \widetilde{M}_{A_{k} B_{k}}^{a_{i}}(z)
$$

совпадают с $M_{A_{k+1} B_{k+1}}^{a_{i}}(z)$ и $T_{a_{i}}$ соответственно.

ЛЕмма 2. Однородная система уравнений, соответствующая системе (9),

$$
\varphi_{A_{k}}^{a_{i}}=-\sum_{d_{k} \neq a_{k}} \sum_{\left(C_{k+1} \neq D_{k+1}\right) \subset a_{k}}^{\prime} M_{A_{k+1} C_{k+1}}^{a_{k}}(z) R_{0}(z) \varphi_{D_{k}}^{a_{i}},
$$

не имеет нетривиальных решений при $\operatorname{Im} z \neq 0$.

Последняя лемма следует из формальной эквивалентности системы (10) уравнению Шредингера [10]

$$
H_{a_{i}} \Psi^{a_{i}}=z \Psi^{a_{i}}, \quad \Psi^{a_{i}}=\sum_{A_{k}} R_{0}(z) \varphi_{A_{k}}^{a_{i}}
$$

Аналогично $T$-матрицам можно определить компоненты резольвент по формулам

$$
\begin{aligned}
R_{a_{N-1} b_{N-1}}^{a_{k}}(z)= & R_{0}(z) \delta_{a_{N-1} b_{N-1}}-R_{0}(z) V_{a_{N-1}} R_{a_{k}}(z) \\
R_{A_{i-1} B_{i-1}}^{a_{k}}(z)= & R_{A_{i+1} B_{i+1}}^{a_{i}}(z) \delta_{a_{i} b_{i}} \delta_{a_{i-1} b_{i-1}}- \\
& -\sum_{\left(d_{i} \neq a_{i}\right) \subset a_{i-1}} \sum_{\left(C_{i+1} \neq D_{i+1}\right) \subset a_{i}}^{\prime} R_{A_{i+1} C_{i+1}}^{a_{i}}(z) V_{c_{N-1}} R_{D_{i} B_{i}}^{a_{k}}(z) .
\end{aligned}
$$

Эти соотношения приводят к уравнениям

$$
\begin{aligned}
R_{A_{k} B_{k}}^{a_{i}}(z)= & R_{A_{k+1} B_{k+1}}^{a_{k}}(z) \delta_{a_{k} b_{k}}- \\
& -\sum_{d_{k} \neq a_{k}} \sum_{\left(C_{k+1} \neq D_{k+1}\right) \subset a_{k}}^{\prime} R_{A_{k+1} C_{k+1}}^{a_{k}}(z) V_{c_{N-1}} R_{D_{k} B_{k}}^{a_{i}}(z) .
\end{aligned}
$$

Данные уравнения будут выведены в разделе 5 настоящей работы. Там же будет доказано свойство

$$
R_{A_{k+1} C_{k+1}}^{a_{k}}(z) V_{c_{N-1}}=R_{0}(z) M_{A_{k+1} C_{k+1}}^{a_{k}}(z),
$$

из которого следует, что

$$
R_{A_{k} B_{k}}^{a_{i}}(z) V_{b_{N-1}}=R_{0}(z) M_{A_{k} B_{k}}^{a_{i}}(z)
$$

для произвольного $a_{i}$. Последняя формула является обобщением на случай многих компонент хорошо известного равенства $R(z) V=R_{0}(z) T(z)$. Равенство (13) позволяет получить связь между компонентами $T$-матрицы и резольвенты:

$$
M_{A_{k} B_{k}}^{a_{i}}(z)=\left(H_{0}-z\right) R_{A_{k} B_{k}}^{a_{i}}(z) V_{b_{N-1}} .
$$


Подобно системе (10) однородная система уравнений, отвечающая (12),

$$
\psi_{A_{k}}^{a_{i}}=-\sum_{d_{k} \neq a_{k}} \sum_{\left(C_{k+1} \neq D_{k+1}\right) \subset a_{k}}^{\prime} R_{A_{k+1} C_{k+1}}^{a_{k}}(z) V_{c_{N-1}} \psi_{D_{k}}^{a_{i}},
$$

не имеет нетривиальных решений при $\operatorname{Im} z \neq 0$. Как и в случае системы $(10)$, данное утверждение является следствием эквивалентности уравнений (14) и уравнения Шредингера

$$
H_{a_{i}} \Psi^{a_{i}}=z \Psi^{a_{i}} .
$$

Компоненты $\psi_{A_{k}}^{a_{i}}$ получаются из $\Psi^{a_{i}}$ с помощью следующих формул:

$$
\begin{aligned}
\psi_{A_{N-1}}^{a_{i}} & =-R_{0}(z) V_{a_{N-1}} \Psi^{a_{i}}, \\
\psi_{A_{j-1}}^{a_{i}} & =\sum_{\left(d_{j} \neq a_{j}\right) \subset a_{j-1}} \sum_{\left(C_{j+1} \neq D_{j+1}\right) \subset a_{j}}^{\prime} R_{A_{j+1} C_{j+1}}^{a_{j}}(z) V_{c_{N-1}} \psi_{D_{j}}^{a_{i}} .
\end{aligned}
$$

При этом

$$
\Psi^{a_{i}}=\sum_{A_{k}} \psi_{A_{k}}^{a_{i}}
$$

Если $\psi_{A_{k}}^{a_{i}}$ суть решения системы (14), то $\Psi^{a_{i}}$ - решение системы $(15)$, и в силу самосопряженности оператора $H_{a_{i}}$ получается, что $\Psi^{a_{i}}=0$ при $\operatorname{Im} z \neq 0$. $\mathrm{B}$ свою очередь, из (16) следует, что все $\psi_{A_{j-1}}^{a_{i}}$ равны нулю.

Решения систем (9) и (12) позволяют восстановить $T$-матрицы и резольвенты по формулам

$$
T_{a_{i}}(z)=\sum_{A_{k}, b_{N-1}} M_{A_{k} B_{k}}^{a_{i}}(z), \quad R_{a_{i}}(z)=\sum_{A_{k}} R_{A_{k} B_{k}}^{a_{i}}(z)
$$

\section{3. Т-МАТРИЦА И РЕЗОЛЬВЕНТА В ИМПУЛЬСНОМ ПРОСТРАНСТВЕ И СОБСТВЕННЫЕ ФУНКЦИИ НЕПРЕРЫВНОГО СПЕКТРА}

Введенные в предыдущем разделе операторы $M_{A_{k} B_{k}}^{a_{i}}(z)$ и $R_{A_{k} B_{k}}^{a_{i}}(z)$ реализуются в импульсном пространстве как интегральные операторы. Соответственно системы уравнений (9) и (12) превращаются в системы интегральных уравнений для ядер входящих в них операторов. Эти системы уравнений позволяют явно описать сингулярности ядер $T$-матрицы и резольвенты в импульсном представлении и определить собственные функции непрерывного спектра [14].

Будем называть интегральный оператор с ядром вида $A_{a_{l}}\left(\mathbf{k}_{a_{l}}, \mathbf{k}_{a_{l}}^{\prime}\right) \delta\left(\mathbf{p}_{a_{l}}-\mathbf{p}_{a_{l}}^{\prime}\right)$, где $A_{a_{l}}\left(\mathbf{k}_{a_{l}}, \mathbf{k}_{a_{l}}^{\prime}\right)$ не имеет особенностей типа $\delta$-функции, оператором, связным в разбиении $a_{l}$, и обозначать его как $A_{a_{l}}^{\mathrm{c}}$. Понятно, что резольвенты $R_{a_{k}}(z)$ и $T$-матрицы $T_{a_{k}}(z)$ не являются связными в $a_{k}$ операторами. Ближайшая наша задача явное описание несвязных частей операторов $R_{a_{k}}(z)$ и $T_{a_{k}}(z)$. Справедливо следующее утверждение. 
Лемма 3. Для связных частей операторов $R_{a_{k}}(z)$ u $T_{a_{k}}(z), 1 \leqslant k \leqslant N-1$, имеют место представления

$$
\begin{aligned}
& R_{a_{k}}^{\mathrm{c}}(z)=R_{a_{k}}(z)-R_{0}(z)-\sum_{i=k+1}^{N-1} \sum_{a_{i}} R_{a_{i}}^{\mathrm{c}}(z), \\
& T_{a_{k}}^{\mathrm{c}}(z)=T_{a_{k}}(z)-\sum_{i=k+1}^{N-1} \sum_{a_{i}} T_{a_{i}}^{\mathrm{c}}(z),
\end{aligned}
$$

əде $T_{a_{N-1}}^{\mathrm{c}}=T_{a_{N-1}}$.

ДокАЗАтЕЛьСтво. Заметим, что достаточно доказать второе из равенств (17), так как первое тогда будет следовать из соотношения

$$
R_{a_{k}}(z)=R_{0}(z)-R_{0}(z) T_{a_{k}}(z) R_{0}(z) .
$$

Формулы (17) можно получить с помощью графической техники [25], однако для полноты изложения мы дадим алгебраическое доказательство, используя основное свойство уравнений (9).

Вместо компонент (8) удобно рассмотреть компоненты $T$-матриц $T_{a_{k}}(z)$ вида

$$
\begin{aligned}
& T_{a_{N-1}}^{a_{k}}(z)=V_{a_{N-1}}-V_{a_{N-1}} R_{a_{k}}(z) V_{a_{N-1}}, \\
& T_{A_{i-1}}^{a_{k}}(z)=-\sum_{\left(d_{i} \neq a_{i}\right) \subset a_{i-1}} \sum_{\left(C_{i+1} \neq D_{i+1}\right) \subset a_{i}}^{\prime} M_{A_{i+1} C_{i+1}}^{a_{i}}(z) R_{0}(z) T_{D_{i}}^{a_{k}}(z),
\end{aligned}
$$

которые удовлетворяют уравнениям [25]

$$
T_{A_{i}}^{a_{k}}(z)=T_{A_{i+1}}^{a_{i}}(z)-\sum_{\left(d_{i} \neq a_{i}\right)} \sum_{\left(C_{i+1} \neq D_{i+1}\right) \subset a_{i}}^{\prime} M_{A_{i+1} C_{i+1}}^{a_{i}}(z) R_{0}(z) T_{D_{i}}^{a_{k}}(z) .
$$

Эти уравнения отличаются от (9) тем, что свободный член $T_{A_{i+1}}^{a_{i}}(z)$ является связным в $a_{i}$ оператором и представляет собой компоненту связной части оператора $T_{a_{i}}(z)$. При этом оператор $T_{a_{i}}^{\mathrm{c}}(z)$ дается формулой

$$
T_{a_{i}}^{\mathrm{c}}(z)=\sum_{A_{i+1}} T_{A_{i+1}}^{a_{i}}(z) .
$$

Из определений (18) и уравнений (19) получаем для $T_{A_{i+1}}^{a_{k}}(z)$ следующее соотношение:

$$
T_{A_{i+1}}^{a_{k}}(z)=T_{A_{i+2}}^{a_{i+1}}(z)+\sum_{a_{i}} T_{A_{i}}^{a_{k}}(z) .
$$

Из свойств уравнений (19), доказанных в работах [10], [11], следует, что второе слагаемое в (19) при $i=k+1$ является связным в $a_{k}$ оператором. Отсюда, с учетом формул (20) и соотношения

$$
T_{a_{k}}(z)=\sum_{a_{N-1}} T_{a_{N-1}}^{a_{k}}(z),
$$

получаем требуемое представление

$$
T_{a_{k}}^{\mathrm{c}}(z)=T_{a_{k}}(z)-\sum_{i=k+1}^{N-1} \sum_{a_{i}} T_{a_{i}}^{\mathrm{c}}(z)
$$

и соответствующее представление для $R_{a_{k}}^{\mathrm{c}}(z)$. Лемма доказана. 
Для описания структуры сингулярностей $T$-матриц нам понадобятся несколько новых обозначений. Пусть $\psi^{a_{i}}\left(\mathbf{k}_{a_{i}}\right)$ - собственная функция дискретного спектра и $\varepsilon_{a_{i}}-$ абсолютная величина отвечающего ей собственного значения $\left(\varepsilon_{a_{i}}>0\right)$ для оператора $h_{a_{i}}$ :

$$
h_{a_{i}} \psi^{a_{i}}\left(\mathbf{k}_{a_{i}}\right)=-\varepsilon_{a_{i}} \psi^{a_{i}}\left(\mathbf{k}_{a_{i}}\right) .
$$

Состояние системы $N$ частиц, в котором взаимодействуют лишь частицы из подсистем разбиения $a_{i}$, описываемое функцией $\psi^{a_{i}}\left(\mathbf{k}_{a_{i}}\right)$, называют $i$-кластерным $к a-$ налом.

Введем формфактор формулой

$$
\phi^{a_{i}}=\sum_{a_{N-1} \subset a_{i}} V_{a_{N-1}} \psi^{a_{i}}
$$

Для $\psi^{a_{i}}, \phi^{a_{i}}$ и $\varepsilon_{a_{i}}$ под индексом $a_{i}$ будем понимать в дальнейшем не только само разбиение $a_{i}$, но и возможные дополнительные индексы (квантовые числа), классифицирующие собственные функции и собственные значения оператора $h_{a_{i}}$. Далее будем считать выполненным условие $\varepsilon_{a_{i}}<\varepsilon_{a_{k}}$ при $a_{i} \subset a_{k}$, согласующееся с известным утверждением [26] о порогах непрерывного спектра для многочастичных гамильтонианов.

$T$-матрица $T(z)$ оператора $H$ описывается формулами [14]

$$
T(z)=\sum_{i=2}^{N-1} \sum_{a_{i}} T_{a_{i}}^{\mathrm{c}}(z)+T^{\mathrm{c}}(z),
$$

где ядра операторов $T_{a_{i}}^{\mathrm{c}}(z)$ при $2 \leqslant i \leqslant N-1$ и оператора $T_{a_{1}}^{\mathrm{c}}(z) \equiv T^{\mathrm{c}}(z)$ задаются формулами (здесь и далее черта означает комплексное сопряжение)

$$
\begin{aligned}
T_{a_{i}}^{\mathrm{c}}\left(\mathbf{P}, \mathbf{P}^{\prime}, z\right)= & t_{a_{i}}^{\mathrm{c}}\left(\mathbf{k}_{a_{i}}, \mathbf{k}_{a_{i}}^{\prime}, z-\mathbf{p}_{a_{i}}^{2}\right) \delta\left(\mathbf{p}_{a_{i}}-\mathbf{p}_{a_{i}}^{\prime}\right), \\
t_{a_{i}}^{\mathrm{c}}\left(\mathbf{k}_{a_{i}}, \mathbf{k}_{a_{i}}^{\prime}, z\right)= & \frac{\phi^{a_{i}}\left(\mathbf{k}_{a_{i}}\right) \bar{\phi}^{a_{i}}\left(\mathbf{k}_{a_{i}}^{\prime}\right)}{z+\varepsilon_{a_{i}}}+ \\
& +\sum_{a_{j}, a_{l} \subset a_{i}} \frac{\phi^{a_{j}}\left(\mathbf{k}_{a_{j}}\right)}{z-\mathbf{p}_{a_{j} a_{i}}^{2}+\varepsilon_{a_{j}}} \mathcal{H}_{a_{j} a_{l}}^{a_{i}}\left(\mathbf{p}_{a_{j} a_{i}}, \mathbf{p}_{a_{l} a_{i}}^{\prime}, z\right) \frac{\bar{\phi}^{a_{l}}\left(\mathbf{k}_{a_{l}}^{\prime}\right)}{z-\mathbf{p}_{a_{l} a_{i}}^{\prime 2}+\varepsilon_{a_{l}}} .
\end{aligned}
$$

В этих формулах $\mathbf{p}_{a_{j} a_{i}}-$ набор относительных импульсов, внешних по отношению к разбиению $a_{j}$ и внутренних по отношению к разбиению $a_{i} \supset a_{j}$, так что

$$
\mathbf{P}=\left\{\mathbf{k}_{a_{i}}, \mathbf{p}_{a_{i}}\right\}=\left\{\mathbf{k}_{a_{j}}, \mathbf{p}_{a_{j}}\right\}, \quad \mathbf{p}_{a_{j}}=\left\{\mathbf{p}_{a_{j} a_{i}}, \mathbf{p}_{a_{i}}\right\}, \quad \mathbf{k}_{a_{i}}=\left\{\mathbf{k}_{a_{j}}, \mathbf{p}_{a_{j} a_{i}}\right\} .
$$

При суммировании по $a_{j}$ и $a_{l}$ во втором уравнении формулы (22) допускаются значения $j, l=N$, при этом полагается

$$
\frac{\phi^{a_{N}}\left(\mathbf{k}_{a_{N}}\right)}{z-\mathbf{p}_{a_{N} a_{i}}^{2}+\varepsilon_{a_{N}}} \equiv 1 .
$$

Доказательство соотношений (22) можно получить по индукции с помощью формул из работы [1] и уравнений (9) и (19). Мы не будем приводить его здесь из-за чрезвычайной громоздкости промежуточных формул, которые частично приведены в статье [2]. 
Кроме явно выделенных в (22) особенностей, называемых главными, функции $t_{a_{i}}^{\mathrm{c}}\left(\mathbf{k}_{a_{i}}, \mathbf{k}_{a_{i}}^{\prime}, z\right)$ имеют ряд второстепенных сингулярностей, отвечающих первым итерациям систем (9) и (19). Эти сингулярности соответствуют законам сохранения энергии в процессах многократных разделенных столкновений кластеров. Отличительной чертой второстепенных сингулярностей является зависимость не только от модулей векторов $\mathbf{p}_{a_{j} a_{i}}$ и $\mathbf{p}_{a_{j} a_{i}}^{\prime}$, но и от углов между этими векторами. Мы также не будем приводить здесь соответствующие формулы из-за их громоздкости [2].

Представление для ядра резольвенты получается из (21) и (22) с помощью формулы

$$
R(z)=R_{0}(z)-R_{0}(z) T(z) R_{0}(z)
$$

Теперь мы имеем возможность определить собственные функции непрерывного спектра оператора $H$. Обозначим через $\chi_{b_{l}}\left(\mathbf{P}^{\prime}, \mathbf{p}_{b_{l}}\right)$ волновую функцию $l$ свободных кластеров. Функция $\chi_{b_{l}}$ выражается через $\psi^{b_{l}}$ с помощью формулы

$$
\chi_{b_{l}}\left(\mathbf{P}^{\prime}, \mathbf{p}_{b_{l}}\right)=\psi^{b_{l}}\left(\mathbf{k}_{b_{l}}\right) \delta\left(\mathbf{p}_{b_{l}}^{\prime}-\mathbf{p}_{b_{l}}\right) .
$$

Собственная функция $\Psi_{b_{l}}^{( \pm)}\left(\mathbf{P}^{\prime}, \mathbf{p}_{b_{l}}\right)$, отвечающая $l$-кластерному начальному $(+)$ или конечному $(-)$ состоянию, определяется как предел

$$
\Psi_{b_{l}}^{( \pm)}=\mp \lim _{\epsilon \rightarrow+0} \mathrm{i} \epsilon R\left(E_{b_{l}} \pm \mathrm{i} \epsilon\right) \chi_{b_{l}}
$$

где энергия $E_{b_{l}}=\mathbf{p}_{b_{l}}^{2}-\varepsilon_{b_{l}}$. Используя соотношения (21)-(23) для вычисления предела в (24), получаем следующую формулу:

$$
\Psi_{b_{l}}^{( \pm)}\left(\mathbf{P}^{\prime}, \mathbf{p}_{b_{l}}\right)=\chi_{b_{l}}\left(\mathbf{P}^{\prime}, \mathbf{p}_{b_{l}}\right)+\sum_{a_{k} \supset b_{l}} u_{a_{k} b_{l}}^{ \pm}\left(\mathbf{k}_{a_{k}}^{\prime}, \mathbf{p}_{b_{l} a_{k}}\right) \delta\left(\mathbf{p}_{a_{k}}^{\prime}-\mathbf{p}_{a_{k}}\right)+u_{b_{l}}^{ \pm}\left(\mathbf{P}^{\prime}, \mathbf{p}_{b_{l}}\right)
$$

где $u_{a_{k} b_{l}}^{ \pm}\left(\mathbf{k}_{a_{k}}^{\prime}, \mathbf{p}_{b_{l} a_{k}}\right)$ и $u_{b_{l}}^{ \pm}\left(\mathbf{P}^{\prime}, \mathbf{p}_{b_{l}}\right)$ - связные части волновых функций $\psi_{a_{k} b_{l}}^{( \pm)}\left(\mathbf{k}_{a_{k}}^{\prime}, \mathbf{p}_{b_{l} a_{k}}\right)$, которые задаются формулами

$$
\begin{aligned}
u_{a_{k} b_{l}}^{ \pm}\left(\mathbf{k}_{a_{k}}^{\prime}, \mathbf{p}_{b_{l} a_{k}}\right)= & -\frac{1}{\mathbf{k}_{a_{k}}^{\prime 2}-E_{b_{l} a_{k}} \mp \mathrm{i} 0} \times \\
& \times \sum_{a_{i} \subset a_{k}} \frac{\phi_{a_{i}}\left(\mathbf{k}_{a_{i}}^{\prime}\right) \mathcal{H}_{a_{i} b_{l}}^{a_{k}}\left(\mathbf{p}_{a_{i} a_{k}}^{\prime}, \mathbf{p}_{b_{l} a_{k}}, E_{b_{l} a_{k}} \pm \mathrm{i} 0\right)}{E_{b_{l} a_{k}}-\mathbf{p}_{a_{i} a_{k}}^{\prime 2}+\varepsilon_{a_{i}} \mp \mathrm{i} 0} \\
u_{b_{l}}^{ \pm}\left(\mathbf{P}^{\prime}, \mathbf{p}_{b_{l}}\right)= & -\frac{1}{\mathbf{P}^{\prime 2}-E_{b_{l}} \mp \mathrm{i} 0} \sum_{a_{i}} \frac{\phi_{a_{i}}\left(\mathbf{k}_{a_{i}}^{\prime}\right) \mathcal{H}_{a_{i} b_{l}}\left(\mathbf{p}_{a_{i}}^{\prime}, \mathbf{p}_{b_{l}}, E_{b_{l}} \pm \mathrm{i} 0\right)}{E_{b_{l}}-\mathbf{p}_{a_{i}}^{\prime 2}+\varepsilon_{a_{i}} \mp \mathrm{i} 0} .
\end{aligned}
$$

Здесь $E_{b_{l} a_{k}}=\mathbf{p}_{b_{l} a_{k}}^{2}-\varepsilon_{b_{l}}$.

Рассмотрим $N$ невзаимодействующих частиц. Такая система описывается волновой функцией

$$
\chi_{0}\left(\mathbf{P}^{\prime}, \mathbf{P}\right)=\delta\left(\mathbf{P}^{\prime}-\mathbf{P}\right) .
$$

Собственная функция, отвечающая системе $N$ частиц, свободных в начальном $(+)$ или конечном $(-)$ состоянии, определяется как предел

$$
\Psi_{0}^{( \pm)}=\mp \lim _{\epsilon \rightarrow+0} \mathrm{i} \epsilon R(E \pm \mathrm{i} \epsilon) \chi_{0}
$$

где энергия $E=\mathbf{P}^{2}$. Вычисляя предел, как и выше, получим

$$
\Psi_{0}^{( \pm)}\left(\mathbf{P}^{\prime}, \mathbf{P}\right)=\chi_{0}\left(\mathbf{P}^{\prime}, \mathbf{P}\right)+\sum_{a_{k}} u_{a_{k} 0}^{ \pm}\left(\mathbf{k}_{a_{k}}^{\prime}, \mathbf{k}_{a_{k}}\right) \delta\left(\mathbf{p}_{a_{k}}^{\prime}-\mathbf{p}_{a_{k}}\right)+u_{0}^{ \pm}\left(\mathbf{P}^{\prime}, \mathbf{P}\right) .
$$


Здесь $u_{a_{k} 0}^{ \pm}\left(\mathbf{k}_{a_{k}}^{\prime}, \mathbf{k}_{a_{k}}\right)$ и $u_{0}^{ \pm}\left(\mathbf{P}^{\prime}, \mathbf{P}\right)$ - связные части волновых функций $\psi_{a_{k} 0}^{( \pm)}\left(\mathbf{k}_{a_{k}}^{\prime}, \mathbf{k}_{a_{k}}\right)$, которые выражаются через ядра $T$-матриц $T_{a_{k}}^{\mathrm{c}}(z)$ с помощью формул

$$
\begin{gathered}
u_{a_{k} 0}^{ \pm}\left(\mathbf{k}_{a_{k}}^{\prime}, \mathbf{k}_{a_{k}}\right)=-\frac{1}{\mathbf{k}_{a_{k}}^{\prime 2}-\mathbf{k}_{a_{k}}^{2} \mp \mathrm{i} 0} t_{a_{k}}^{\mathrm{c}}\left(\mathbf{k}_{a_{k}}^{\prime}, \mathbf{k}_{a_{k}}, \mathbf{k}_{a_{k}}^{2} \pm \mathrm{i} 0\right), \\
u_{0}^{ \pm}\left(\mathbf{P}^{\prime}, \mathbf{P}\right)=-\frac{1}{\mathbf{P}^{\prime 2}-\mathbf{P}^{2} \mp \mathrm{i} 0} T^{\mathrm{c}}\left(\mathbf{P}^{\prime}, \mathbf{P}, \mathbf{P}^{2} \pm \mathrm{i} 0\right) .
\end{gathered}
$$

\section{4. РЕЗОЛЬВЕНТА И СОБСТВЕННЫЕ ФУНКЦИИ НЕПРЕРЫВНОГО СПЕКТРА В КОНФИГУРАЦИОННОМ ПРОСТРАНСТВЕ}

В данном разделе мы опишем асимптотические свойства ядра резольвенты оператора $H$ и волновых функций $\Psi_{b_{l}}^{( \pm)}$в конфигурационном пространстве. Ядро резольвенты $R(z)$ в конфигурационном пространстве дается преобразованием Фурье:

$$
R\left(\mathbf{X}, \mathbf{X}^{\prime}, z\right)=\frac{1}{(2 \pi)^{d}} \int d \mathbf{P} d \mathbf{P}^{\prime} e^{\mathrm{i}(\mathbf{P}, \mathbf{X})} R\left(\mathbf{P}, \mathbf{P}^{\prime}, z\right) e^{-\mathrm{i}\left(\mathbf{P}^{\prime}, \mathbf{X}^{\prime}\right)},
$$

где $R\left(\mathbf{P}, \mathbf{P}^{\prime}, z\right)$ - ядро оператора $R(z)$ в импульсном представлении. Используя связь (23) операторов $R(z)$ и $T(z)$, представим ядро $R\left(\mathbf{X}, \mathbf{X}^{\prime}, z\right)$ в виде

$$
R\left(\mathbf{X}, \mathbf{X}^{\prime}, z\right)=R_{0}\left(\mathbf{X}, \mathbf{X}^{\prime}, z\right)+G\left(\mathbf{X}, \mathbf{X}^{\prime}, z\right) .
$$

Здесь $R_{0}\left(\mathbf{P}, \mathbf{P}^{\prime}, z\right)$ - ядро оператора $R_{0}(z)$, которое выражается через функцию Ханкеля:

$$
R_{0}\left(\mathbf{X}, \mathbf{X}^{\prime}, z\right)=\frac{\mathrm{i}}{4}\left(\frac{\sqrt{z}}{2 \pi}\right)^{(n-2) / 2} \frac{H_{(n-2) / 2}^{(1)}\left(\sqrt{z}\left|\mathbf{X}-\mathbf{X}^{\prime}\right|\right)}{\left|\mathbf{X}-\mathbf{X}^{\prime}\right|^{(n-2) / 2}} .
$$

Ядро $G\left(\mathbf{X}, \mathbf{X}^{\prime}, z\right)$ дается преобразованием Фурье (30) от функции

$$
g\left(\mathbf{P}, \mathbf{P}^{\prime}, z\right)=-\frac{T\left(\mathbf{P}, \mathbf{P}^{\prime}, z\right)}{\left(\mathbf{P}^{2}-z\right)\left(\mathbf{P}^{\prime 2}-z\right)} .
$$

Следует отметить, что в силу представления (31) ядро $R\left(\mathbf{X}, \mathbf{X}^{\prime}, z\right)$ имеет стандартную сингулярность при $\mathbf{X}=\mathbf{X}^{\prime}$ :

$$
R\left(\mathbf{X}, \mathbf{X}^{\prime}, z\right)=\frac{1}{(d-2) \sigma_{d}\left|\mathbf{X}-\mathbf{X}^{\prime}\right|^{d-2}}\left(1+O\left(\left|\mathbf{X}-\mathbf{X}^{\prime}\right|\right),\right.
$$

где $\sigma_{d}$ - площадь единичной сферы в $\mathbb{R}^{d}$.

Опишем асимптотический вид ядра $R\left(\mathbf{X}, \mathbf{X}^{\prime}, z\right)$ при $\left|\mathbf{X}^{\prime}\right| \rightarrow \infty$ и $|\mathbf{X}|=O\left(\left|\mathbf{X}^{\prime}\right|^{\nu}\right)$, $\nu<1$. Поскольку $R(z)$ как функция от $z$ имеет разрез на вещественной оси, следует, как обычно, различать пределы при $z \rightarrow E \pm \mathrm{i} 0$ (здесь $\operatorname{Im} E=0$ ). Для вычисления асимптотики ядер $R\left(\mathbf{X}, \mathbf{X}^{\prime}, E \pm \mathrm{i} 0\right)$ воспользуемся полученой в разделе 3 структурой сингулярностей ядра резольвенты в импульсном пространстве и представлением (30).

Докажем следующее утверждение.

Теорема 1. Ядро $R\left(\mathbf{X}, \mathbf{X}^{\prime}, E \pm \mathrm{i} 0\right)$ представляется в виде

$$
R\left(\mathbf{X}, \mathbf{X}^{\prime}, E \pm \mathrm{i} 0\right)=\sum_{b_{l}} \bar{\psi}_{b_{l}}\left(\mathbf{x}_{b_{l}}^{\prime}\right) Q_{b_{l}}^{ \pm}\left(\mathbf{X}, \mathbf{y}_{b_{l}}^{\prime}\right)+Q_{0}^{ \pm}\left(\mathbf{X}, \mathbf{X}^{\prime}\right)
$$


где функиии $Q_{b_{l}}^{ \pm}\left(\mathbf{X}, \mathbf{y}_{b_{l}}^{\prime}\right)$ и $Q_{0}^{ \pm}\left(\mathbf{X}, \mathbf{X}^{\prime}\right)$ при $\left|\mathbf{y}_{b_{l}}^{\prime}\right| \rightarrow \infty u\left|\mathbf{X}^{\prime}\right| \rightarrow \infty$ переходят в сферические волны:

$$
\begin{aligned}
Q_{b_{l}}^{ \pm}\left(\mathbf{X}, \mathbf{y}_{b_{l}}^{\prime}\right) \sim & \frac{1}{4 \pi}\left(\frac{\sqrt{E}}{2 \pi}\right)^{\left(d_{l}-3\right) / 2} \Psi_{b_{l}}^{( \pm)}\left(\mathbf{X}, \mp \sqrt{E+\varepsilon_{b_{l}}} \hat{\mathbf{y}}_{b_{l}}^{\prime}\right) \times \\
& \times\left|\mathbf{y}_{b_{l}}^{\prime}\right|^{\left(d_{l}-1\right) / 2} \exp \left\{ \pm \mathrm{i} \sqrt{E+\varepsilon_{b_{l}}}\left|\mathbf{y}_{b_{l}}^{\prime}\right| \mp \mathrm{i} \frac{\pi\left(d_{l}-3\right)}{4}\right\}, \\
Q_{0}^{ \pm}\left(\mathbf{X}, \mathbf{X}^{\prime}\right) \sim & \frac{1}{4 \pi}\left(\frac{\sqrt{E}}{2 \pi}\right)^{(d-3) / 2} \Psi_{0}^{( \pm)}\left(\mathbf{X}, \mp \sqrt{E} \widehat{\mathbf{X}}^{\prime}\right) \times \\
& \times\left|\mathbf{X}^{\prime}\right|^{(d-1) / 2} \exp \left\{ \pm \mathrm{i} \sqrt{E}\left|\mathbf{X}^{\prime}\right| \mp \mathrm{i} \frac{\pi(d-3)}{4}\right\} .
\end{aligned}
$$

Здесъ $d_{l}=3(l-1), d=3(N-1)$, а функции $\Psi_{b_{l}}^{( \pm)}\left(\mathbf{X}, \mathbf{p}_{b_{l}}\right)$ и $\Psi_{0}^{( \pm)}(\mathbf{X}, \mathbf{P})$ являются собственными функииями непрерывного спектра оператора $H$ в конфигурачионном пространстве и связаны с бункииями (25) и (28) преобразованием Фуръе, например

$$
\Psi_{b_{l}}^{( \pm)}\left(\mathbf{X}, \mathbf{p}_{b_{l}}^{\prime}\right)=\frac{1}{\sqrt{(2 \pi)^{d-d_{l}}}} \int d \mathbf{P} \Psi_{b_{l}}^{( \pm)}\left(\mathbf{P}, \mathbf{p}_{b_{l}}^{\prime}\right) e^{\mathrm{i}(\mathbf{P}, \mathbf{X})} .
$$

ДокАзАтЕльство. Для определенности рассмотрим случай ядра $R\left(\mathbf{X}, \mathbf{X}^{\prime}, E^{+}\right)$, $E^{+}=E+\mathrm{i} 0$. Запишем формулу $(23)$ в терминах ядер участвующих в ней операторов:

$$
R\left(\mathbf{P}, \mathbf{P}^{\prime}, E^{+}\right)=\frac{\delta\left(\mathbf{P}-\mathbf{P}^{\prime}\right)}{\mathbf{P}^{2}-E^{+}}-\frac{T\left(\mathbf{P}, \mathbf{P}^{\prime}, E^{+}\right)}{\left(\mathbf{P}^{2}-E^{+}\right)\left(\mathbf{P}^{\prime 2}-E^{+}\right)} .
$$

Ядро $T\left(\mathbf{P}, \mathbf{P}^{\prime}, E^{+}\right)$представим в виде

$$
T\left(\mathbf{P}, \mathbf{P}^{\prime}, E^{+}\right)=\sum_{b_{l}} u_{b_{l}}\left(\mathbf{P}, \mathbf{p}_{b_{l}}^{\prime}, E^{+}\right) \frac{\bar{\phi}_{b_{l}}\left(\mathbf{k}_{b_{l}}^{\prime}\right)}{E^{+}-\mathbf{p}_{b_{l}}^{\prime 2}+\varepsilon_{b_{l}}},
$$

где

$$
\begin{aligned}
u_{b_{l}}\left(\mathbf{P}, \mathbf{p}_{b_{l}}^{\prime}, E^{+}\right)= & \phi_{b_{l}}\left(\mathbf{k}_{b_{l}}\right) \delta\left(\mathbf{p}_{b_{l}}-\mathbf{p}_{b_{l}}^{\prime}\right)+ \\
& +\sum_{a_{k} \supset a_{i}, b_{l}} \frac{\phi_{a_{i}}\left(\mathbf{k}_{a_{i}}\right)}{E^{+}-\mathbf{p}_{a_{i}}^{2}+\varepsilon_{a_{i}}} \mathcal{H}_{a_{i} b_{l}}^{a_{k}}\left(\mathbf{p}_{a_{i} a_{k}}, \mathbf{p}_{b_{l} a_{k}}^{\prime}, E^{+}-\mathbf{p}_{a_{k}}^{\prime 2}\right) \delta\left(\mathbf{p}_{a_{k}}-\mathbf{p}_{a_{k}}^{\prime}\right) .
\end{aligned}
$$

Заметим, что при $E=\mathbf{p}_{b_{l}}^{\prime 2}-\varepsilon_{b_{l}}$ функция $\left(\mathbf{P}^{2}-E^{+}\right)^{-1} u_{b_{l}}$ совпадает с волновой функцией $\Psi_{b_{l}}^{+}$. Подставляя представление (34) в (30), получаем

$$
R\left(\mathbf{X}, \mathbf{X}^{\prime}, E^{+}\right)=\frac{1}{(2 \pi)^{d}} \int d \mathbf{P}^{\prime} \widehat{R}\left(\mathbf{X}, \mathbf{P}^{\prime}, E^{+}\right) e^{\mathrm{i}\left(\mathbf{P}^{\prime},-\mathbf{X}^{\prime}\right)},
$$

где через $\widehat{R}\left(\mathbf{X}, \mathbf{P}^{\prime}, E^{+}\right)$обозначено преобразование Фурье ядра $R\left(\mathbf{P}, \mathbf{P}^{\prime}, E^{+}\right)$по первому аргументу. Учтем далее, что особенности сингулярных знаменателей $\mathbf{P}^{\prime 2}-E^{+}$ и $E^{+}-\mathbf{p}_{b_{l}}^{\prime 2}+\varepsilon_{b_{l}}$ не пересекаются:

$$
\mathbf{P}^{\prime 2}-E^{+}+E^{+}-\mathbf{p}_{b_{l}}^{\prime 2}+\varepsilon_{b_{l}}=\mathbf{k}_{b_{l}}^{\prime 2}+\varepsilon_{b_{l}}>0 .
$$

Выделим из области интегрирования в (36) окрестность $\Omega$ гиперсферы $\mathbf{P}^{\prime 2}=E$ такую, что в ней знаменатели $E^{+}-\mathbf{p}_{b_{l}}^{\prime 2}+\varepsilon_{b_{l}}$ не обращаются в нуль. В этой области 
подынтегральная функция в (36) имеет единственную особенность $\left(\mathbf{P}^{\prime 2}-E^{+}\right)^{-1}$. Асимптотика интеграла (36), взятого по выделенной области, легко вычисляется с помощью формулы [2]

$$
\begin{aligned}
I(\mathbf{x}, E)= & \int d q e^{\mathrm{i}(\mathbf{x}, \mathbf{q})} \frac{f(\mathbf{q})}{\mathbf{q}^{2}-E \mp \mathrm{i} 0}= \\
= & \left(\frac{2 \pi}{|\mathbf{x}|}\right)^{(n-1) / 2} \pi E^{(n-3) / 4} \exp \left\{ \pm \mathrm{i} \sqrt{E}|\mathbf{x}| \mp \mathrm{i} \frac{(n-3) \pi}{4}\right\} \times \\
& \times\left[f( \pm \sqrt{E} \hat{\mathbf{x}})+O\left(\frac{1}{\sqrt{E}|\mathbf{x}|}\right)\right], \quad \mathbf{x}, \mathbf{q} \in \mathbb{R}^{n} .
\end{aligned}
$$

Применяя эту асимптотику к интегралу (36), взятому по области $\Omega$, получаем в старшем порядке выражение для $Q_{0}^{+}$из $(33)$.

Рассмотрим интеграл (36) по оставшейся части пространства $\mathbb{R}^{d}$. Теперь можно воспользоваться представлением (35). В результате интеграл представляется как ряд слагаемых вида

$$
J\left(\mathbf{X}^{\prime}, E\right)=\frac{1}{(2 \pi)^{d}} \int_{\mathbb{R}^{d} \backslash \Omega} d \mathbf{P}^{\prime} f\left(\mathbf{p}_{b_{l}}^{\prime}\right) \frac{\bar{\phi}_{b_{l}}\left(\mathbf{k}_{b_{l}}^{\prime}\right) e^{\mathrm{i}\left(\mathbf{P}^{\prime}, \mathbf{X}^{\prime}\right)}}{\left(E^{+}-\mathbf{p}_{b_{l}}^{\prime 2}+\varepsilon_{b_{l}}\right)\left(\mathbf{P}^{\prime 2}-E^{+}\right)},
$$

причем в области $\mathbb{R}^{d} \backslash \Omega$ выражение $\mathbf{P}^{\prime 2}-E$ не обращается в нуль. Для вычисления асимптотики $J\left(\mathbf{X}^{\prime}, E\right)$ при $\left|\mathbf{X}^{\prime}\right| \rightarrow \infty$ проведем сначала интегрирование по переменной $\mathbf{p}_{b_{l}}^{\prime}$ с помощью формулы (37) и учтем, что

$$
\frac{1}{\sqrt{(2 \pi)^{d-d_{l}}}} \int d \mathbf{k}_{b_{l}}^{\prime} e^{\mathrm{i}\left(\mathbf{x}_{b_{l}}^{\prime}, \mathbf{k}_{b_{l}}^{\prime}\right)} \frac{\bar{\phi}_{b_{l}}\left(\mathbf{k}_{b_{l}}^{\prime}\right)}{\mathbf{k}_{b_{l}}^{\prime 2}+\varepsilon_{b_{l}}}=\bar{\psi}_{b_{l}}\left(\mathbf{x}_{b_{l}}^{\prime}\right)
$$

Тогда в старшем порядке интеграл $J\left(\mathbf{X}^{\prime}, E\right)$ дается выражением

$$
\begin{aligned}
J\left(\mathbf{X}^{\prime}, E\right) \sim & \frac{1}{4 \pi \sqrt{(2 \pi)^{d-d_{l}}}}\left(\frac{\sqrt{E+\varepsilon_{b_{l}}}}{2 \pi}\right)^{\left(d_{l}-3\right) / 2} \bar{\psi}_{b_{l}}\left(\mathbf{x}_{b_{l}}^{\prime}\right) f\left(\sqrt{E+\varepsilon_{b_{l}}} \hat{\mathbf{y}}_{b_{l}}^{\prime}\right) \times \\
& \times\left|\mathbf{y}_{b_{l}}^{\prime}\right|^{\left(d_{l}-1\right) / 2} \exp \left\{\mathrm{i} \sqrt{E+\varepsilon_{b_{l}}}\left|\mathbf{y}_{b_{l}}^{\prime}\right|-\mathrm{i} \frac{\pi\left(d_{l}-3\right)}{4}\right\} .
\end{aligned}
$$

Собирая полученные вклады, получаем для ядра $R\left(\mathbf{X}, \mathbf{X}^{\prime}, E^{+}\right)$требуемое представление (32). Теорема доказана.

Перейдем к обсуждению асимптотических свойств волновых функций

$$
\Psi_{b_{l}}^{(+)}\left(\mathbf{X}, \mathbf{p}_{b_{l}}\right)=\frac{1}{\sqrt{(2 \pi)^{d-d_{l}}}} \int d \mathbf{P}^{\prime} \Psi_{b_{l}}^{(+)}\left(\mathbf{P}^{\prime}, \mathbf{p}_{b_{l}}\right) e^{\mathrm{i}\left(\mathbf{P}^{\prime}, \mathbf{X}\right)} .
$$

Опишем асимптотику волновых функций $\Psi_{b_{2}}^{(+)}\left(\mathbf{X}, \mathbf{p}_{b_{2}}\right)$, которые отвечают двукластерным начальным состояниям. В этом случае можно показать, что функции $\Psi_{b_{2}}^{(+)}\left(\mathbf{P}, \mathbf{p}_{b_{2}}\right)$ не содержат второстепенных сингулярностей, связанных с перерассеянием кластеров. Это означает, что амплитуды в формулах (26) являются гладкими функциями. Последнее позволяет легко вычислить асимптотику волновых функций $\Psi_{b_{2}}^{(+)}\left(\mathbf{X}, \mathbf{p}_{b_{2}}\right)$ при $|\mathbf{X}| \rightarrow \infty$, используя приемы, аналогичные тем, что применялись при доказательстве теоремы 1. Приведем окончательный результат. 
Функции $\Psi_{b_{2}}^{(+)}\left(\mathbf{X}, \mathbf{p}_{b_{2}}\right)$ представляются в виде

$$
\Psi_{b_{2}}^{(+)}\left(\mathbf{X}, \mathbf{p}_{b_{2}}\right)=\chi_{b_{2}}\left(\mathbf{X}, \mathbf{p}_{b_{2}}\right)+\sum_{i=2}^{N} \sum_{a_{i}} \psi_{a_{i}}\left(\mathbf{x}_{a_{i}}\right) U_{a_{i} b_{2}}^{+}\left(\mathbf{y}_{a_{i}}, \mathbf{p}_{b_{2}}\right)
$$

Здесь

$$
\chi_{b_{2}}\left(\mathbf{X}, \mathbf{p}_{b_{2}}\right)=\psi_{b_{2}}\left(\mathbf{x}_{b_{2}}\right) e^{\mathrm{i}\left(\mathbf{p}_{b_{2}}, \mathbf{y}_{b_{2}}\right)},
$$

а функции $U_{a_{i} b_{2}}^{+}\left(\mathbf{y}_{a_{i}}, \mathbf{p}_{b_{2}}\right)$ переходят в сферические волны при $\left|\mathbf{y}_{a_{i}}\right| \rightarrow \infty$ :

$$
\begin{aligned}
U_{a_{i} b_{2}}^{+}\left(\mathbf{y}_{a_{i}}, \mathbf{p}_{b_{2}}\right) \sim & C_{3(i-1)}\left(E_{a_{i} b_{2}}\right) \mathcal{H}_{a_{i} b_{2}}\left(\sqrt{E_{a_{i} b_{2}}} \hat{\mathbf{y}}_{a_{i}}, \mathbf{p}_{b_{2}}, E_{b_{2}}+\mathrm{i} 0\right) \times \\
& \times\left|\mathbf{y}_{a_{i}}\right|^{-(3 i-4) / 2} e^{\mathrm{i} \sqrt{E_{a_{i} b_{2}}}\left|\mathbf{y}_{a_{i}}\right|},
\end{aligned}
$$

где

$$
E_{a_{i} b_{2}}=E_{b_{2}}+\varepsilon_{a_{i}}=\mathbf{p}_{b_{2}}^{2}-\varepsilon_{b_{2}}+\varepsilon_{a_{i}}, \quad C_{m}(E)=-2 \pi^{2} E^{(m-3) / 4} e^{-\mathrm{i} \pi(m-3) / 4} .
$$

В формулах (38) и (39) мы полагаем $\psi_{a_{N}}=1, \varepsilon_{a_{N}}=0$ при $i=N$.

\section{5. ДИФФЕРЕНЦИАЛЬНЫЕ УРАВНЕНИЯ ДЛЯ КОМПОНЕНТ}

В разделе 2 мы ввели компоненты резольвенты $R_{A_{k} B_{k}}^{a_{i}}(z)$, выписали уравнения (12) для этих компонент и анонсировали перестановочное свойство (13). В настоящем разделе мы дадим соответствующие доказательства. Более того, мы покажем, что компоненты резольвенты $R_{A_{k} B_{k}}^{a_{i}}(z)$ являются матричными элементами резольвенты некоторого матричного оператора. Мы построим этот оператор и на его основе получим так называемые дифференциальные уравнения для компонент резольвенты и компонент волновых функций системы $N$ тел.

В дальнейшем удобно использовать матричные обозначения. Сопоставим компонентам $R_{A_{k} B_{k}}^{a_{i}}(z)$ и $M_{A_{k} B_{k}}^{a_{i}}(z)$ матрицы $\mathbf{R}_{k}^{a_{i}}(z)$ и $\mathbf{M}_{k}^{a_{i}}(z)$, столбцы и строки которых нумеруются цепочками разбиений $A_{k}, B_{k}$ такими, что $A_{k}, B_{k} \subset a_{i}$. Определим матрицы $\mathbf{R}_{k j}^{a_{i}}(z), \mathbf{M}_{k j}^{a_{i}}(z), \mathbf{V}_{k}^{a_{i}}, \mathbf{R}_{0 k}^{a_{i}}(z)$ и $\mathbf{I}_{k}^{a_{i}}$ формулами

$$
\begin{aligned}
{\left[\mathbf{R}_{k j}^{a_{i}}(z)\right]_{A_{k} B_{k}} } & =R_{A_{j+1} B_{j+1}}^{a_{j}}(z) \delta_{a_{j} b_{j}} \ldots \delta_{a_{k} b_{k}}, \\
{\left[\mathbf{M}_{k j}^{a_{i}}(z)\right]_{A_{k} B_{k}} } & =M_{A_{j+1} B_{j+1}}^{a_{j}}(z) \delta_{a_{j} b_{j}} \ldots \delta_{a_{k} b_{k}}, \\
{\left[\mathbf{V}_{k}^{a_{i}}\right]_{A_{k} B_{k}} } & =V_{a_{N-1} \delta_{a_{N-1} b_{N-1}} \ldots \delta_{a_{k} b_{k}},} \\
{\left[\mathbf{R}_{0 k}^{a_{i}}(z)\right]_{A_{k} B_{k}} } & =R_{0}(z) \delta_{a_{N-1} b_{N-1}} \ldots \delta_{a_{k} b_{k}}, \\
{\left[\mathbf{I}_{k}^{a_{i}}\right]_{A_{k} B_{k}} } & =I \delta_{a_{N-1} b_{N-1}} \ldots \delta_{a_{k} b_{k}} .
\end{aligned}
$$

Введем числовую матрицу $\mathbf{X}_{k j}^{a_{i}}$, с помощью которой можно учесть ограничения при суммировании в уравнениях (9) и (12):

$$
\begin{gathered}
{\left[\mathbf{X}_{k j}^{a_{i}}\right]_{A_{k} B_{k}}=X_{A_{j+1} B_{j+1}} \delta_{a_{j} b_{j}} \ldots \delta_{a_{k} b_{k}},} \\
X_{A_{j+1} B_{j+1}}=\delta^{a_{j} b_{j}} \delta\left(b_{j+1} \subset a_{j}\right) \ldots \delta\left(b_{N-1} \subset a_{N-2}\right) \delta^{a_{N-1} b_{N-1}} .
\end{gathered}
$$

Здесь $\delta^{a b}=1-\delta_{a b}$, а символ $\delta\left(b_{k+1} \subset a_{k}\right)$ понимается в следующем смысле:

$$
\delta\left(b_{k+1} \subset a_{k}\right)= \begin{cases}1, & b_{k+1} \subset a_{k}, \\ 0, & b_{k+1} \not \subset a_{k} .\end{cases}
$$


Уравнения (9) и (12) в матричной форме принимают вид

$$
\begin{aligned}
\mathbf{M}_{k}^{a_{i}}(z) & =\mathbf{M}_{k k}^{a_{i}}(z)-\mathbf{M}_{k k}^{a_{i}}(z) \mathbf{R}_{0 k}^{a_{i}}(z) \mathbf{X}_{k k}^{a_{i}} \mathbf{M}_{k}^{a_{i}}(z), \\
\mathbf{R}_{k}^{a_{i}}(z) & =\mathbf{R}_{k k}^{a_{i}}(z)-\mathbf{R}_{k k}^{a_{i}}(z) \mathbf{V}_{k}^{a_{i}} \mathbf{X}_{k k}^{a_{i}} \mathbf{R}_{k}^{a_{i}}(z) .
\end{aligned}
$$

Запишем также уравнения для матриц $\mathbf{R}_{k j}^{a_{i}}(z)$, получающиеся из определения этих операторов и уравнений (41):

$$
\mathbf{R}_{k j}^{a_{i}}(z)=\mathbf{R}_{k, j+1}^{a_{i}}(z)-\mathbf{R}_{k, j+1}^{a_{i}}(z) \mathbf{V}_{k}^{a_{i}} \mathbf{X}_{k, j+1}^{a_{i}} \mathbf{R}_{k j}^{a_{i}}(z)
$$

(здесь и далее в двойных нижних индексах использована запятая, когда один из индексов дается выражением вида $j+1$ или $j-1)$.

Нашей первой задачей будет доказательство справедливости уравнения (42) для любого фиксированного $k$ из промежутка $i+1 \leqslant k \leqslant N-1$ и любого $j$ такого, что $k \leqslant j \leqslant N-1$. Рассмотрим уравнение (42) при $j=N-1$. В этом случае оно сводится к виду

$$
R_{a_{N-1}}(z)=R_{0}(z)-R_{0}(z) V_{a_{N-1}} R_{a_{N-1}}(z), \quad R_{a_{N-1}}(z)=\left(H_{0}+V_{a_{N-1}}-z I\right)^{-1},
$$

и представляет собой уравнение Липпманна-Швингера для двухчастичной резольвенты. Таким образом, уравнение (42) справедливо при $j=N-1$. Предположим, что оно верно на $j$-м шаге и докажем его справедливость на $(j-1)$-м шаге. Оператор $\mathbf{R}_{k, j-1}^{a_{i}}(z)$ имеет матричные элементы

$$
\left[\mathbf{R}_{k, j-1}^{a_{i}}(z)\right]_{A_{k} B_{k}}=R_{A_{j} B_{j}}^{a_{j-1}}(z) \delta_{a_{j-1} b_{j-1}} \ldots \delta_{a_{k} b_{k}} .
$$

В свою очередь, компоненты $R_{A_{j} B_{j}}^{a_{j-1}}(z)$ определяются формулой

$$
\begin{aligned}
R_{A_{j} B_{j}}^{a_{j-1}}(z)= & R_{A_{j+2} B_{j+2}}^{a_{j+2}}(z) \delta_{a_{j+1} b_{j+1}} \delta_{a_{j} b_{j}}- \\
& -\sum_{\left(d_{j+1} \neq a_{j+1}\right) \subset a_{j}} \sum_{\left(C_{j+2} \neq D_{j+2}\right) \subset a_{j+1}}^{\prime} R_{A_{j+2} C_{j+2}}^{a_{j+2}}(z) V_{c_{N-1}} R_{D_{j+1} B_{j+1}}^{a_{j-1}}(z) .
\end{aligned}
$$

Суммируя данные равенства по $a_{j}$, используя правило [10], [14]

$$
\sum_{a_{j}} \sum_{\left(d_{j+1} \neq a_{j+1}\right) \subset a_{j}}(\cdot)=\sum_{d_{j+1} \neq a_{j+1}}(\cdot)
$$

и уравнения (42) на $j$-м шаге, приходим к заключению, что

$$
\sum_{a_{j}} R_{A_{j} B_{j}}^{a_{j-1}}(z)=R_{A_{j+1} B_{j+1}}^{a_{j-1}}(z) .
$$

Подставляя это соотношение в правую часть равенства (43), получаем уравнения для компонент $R_{A_{j} B_{j}}^{a_{j-1}}(z)$, которые в матричной форме имеют вид

$$
\left(\mathbf{X}_{k}^{a_{i}}+\mathbf{R}_{k, j+1}^{a_{i}}(z) \mathbf{V}_{k}^{a_{i}} \mathbf{X}_{k, j+1}^{a_{i}}\right) \mathbf{R}_{k, j-1}^{a_{i}}(z)=\mathbf{R}_{k, j+1}^{a_{i}}(z)-\mathbf{R}_{k, j+1}^{a_{i}}(z) \mathbf{V}_{k}^{a_{i}} \mathbf{X}_{k j}^{a_{i}} \mathbf{R}_{k, j-1}^{a_{i}}(z) .
$$

Отсюда с учетом уравнений (42), записанных в форме

$$
\left(\mathbf{X}_{k}^{a_{i}}+\mathbf{R}_{k, j+1}^{a_{i}}(z) \mathbf{V}_{k}^{a_{i}} \mathbf{X}_{k, j+1}^{a_{i}}\right)^{-1} \mathbf{R}_{k, j+1}^{a_{i}}(z)=\mathbf{R}_{k j}^{a_{i}}(z),
$$

получаем искомые уравнения на $(j-1)$-м шаге:

$$
\mathbf{R}_{k, j-1}^{a_{i}}(z)=\mathbf{R}_{k j}^{a_{i}}(z)-\mathbf{R}_{k j}^{a_{i}}(z) \mathbf{V}_{k}^{a_{i}} \mathbf{X}_{k j}^{a_{i}} \mathbf{R}_{k, j-1}^{a_{i}}(z) .
$$

Это завершает доказательство справедливости уравнений (42). 
Уравнения (41) для операторов $\mathbf{R}_{k}^{a_{i}}(z)$ формально получаются из уравнений (42) при $j=k-1$ с отождествлением $\mathbf{R}_{k, k-1}^{a_{i}}(z)=\mathbf{R}_{k}^{a_{i}}(z)$. Однако уравнения (41) можно получить и независимо от (42), повторяя практически дословно этапы вывода уравнений (42). Чтобы избежать повторения, мы не будем приводить здесь это доказательство, и справедливость уравнений (41) будем считать доказанной.

На следующем этапе мы построим операторы, для которых $\mathbf{R}_{k j}^{a_{i}}(z)$ и $\mathbf{R}_{k}^{a_{i}}(z)$ являются резольвентами.

5.1. Дифференциальные уравнения для компонент резольвенты. Рассмотрим оператор

$$
\mathbf{H}_{k j}^{a_{i}}=H_{0} \mathbf{I}_{k}^{a_{i}}+\mathbf{V}_{k}^{a_{i}}+\mathbf{V}_{k}^{a_{i}} \sum_{m=j+1}^{N-1} \mathbf{X}_{k m}^{a_{i}} .
$$

В координатном представлении в силу равенства $H_{0}=-\Delta_{\mathbf{x}}$ оператор $\mathbf{H}_{k j}^{a_{i}}$ является матричным дифференциальным оператором, действующим на вектор-функции, компоненты которых классифицируются цепочками разбиений $A_{k}$. Справедливо следующее утверждение.

Лемма 4. Оператор $\mathbf{R}_{k j}^{a_{i}}(z)$ является резольвентой оператора $\mathbf{H}_{k j}^{a_{i}}$ :

$$
\left(\mathbf{H}_{k j}^{a_{i}}-z \mathbf{I}_{k}^{a_{i}}\right) \mathbf{R}_{k j}^{a_{i}}(z)=\mathbf{I}_{k}^{a_{i}} .
$$

ДокАЗАТЕЛьство. При $j=N-1$ уравнение (45) принимает вид

$$
\left(H_{0}+V_{a_{N-1}}-z I\right) R_{a_{N-1}}(z)=I,
$$

и тем самым в силу определения $R_{a_{N-1}}(z)=\left(H_{0}+V_{a_{N-1}}-z I\right)^{-1}$ равенство (45) справедливо при $j=N-1$. Предположим, что равенство (45) выполнено на $j$-м шаге. Рассмотрим оператор $\mathbf{R}_{k, j-1}^{a_{i}}(z)$, удовлетворяющий уравнению (42) при замене $j \rightarrow j-1$. Применяя оператор $\mathbf{H}_{k j}^{a_{i}}-z \mathbf{I}_{k}^{a_{i}}$ к обеим частям данного уравнения, получим

$$
\left(\mathbf{H}_{k j}^{a_{i}}-z \mathbf{I}_{k}^{a_{i}}\right) \mathbf{R}_{k, j-1}^{a_{i}}(z)=\mathbf{I}_{k}^{a_{i}}-\mathbf{V}_{k}^{a_{i}} \mathbf{X}_{k j}^{a_{i}} \mathbf{R}_{k, j-1}^{a_{i}}(z),
$$

что совпадает с уравнением $(45)$ на $(j-1)$-м шаге. Тем самым мы показали справедливость уравнения (45) при любом $j$. Лемма доказана.

Найдем теперь оператор, для которого $\mathbf{R}_{k}^{a_{i}}(z)$ является резольвентой. Рассмотрим оператор $\mathbf{H}_{k}^{a_{i}}=\mathbf{H}_{k k}^{a_{i}}+\mathbf{V}_{k}^{a_{i}} \mathbf{X}_{k k}^{a_{i}}$.

Лемма 5. Оператор $\mathbf{R}_{k}^{a_{i}}(z)$ является резольвентой оператора $\mathbf{H}_{k}^{a_{i}}$ :

$$
\left(\mathbf{H}_{k}^{a_{i}}-z \mathbf{I}_{k}^{a_{i}}\right) \mathbf{R}_{k}^{a_{i}}(z)=\mathbf{I}_{k}^{a_{i}} .
$$

ДокАЗАТЕльство. Формула (46) немедленно следует из уравнений (41) и леммы 4 при $j=k$.

Нам осталось доказать сформулированное в разделе 2 коммутационное свойство

$$
R_{A_{k} B_{k}}^{a_{i}}(z) V_{b_{N-1}}=R_{0}(z) M_{A_{k} B_{k}}^{a_{i}}(z),
$$

которое в матричной форме принимает вид

$$
\mathbf{R}_{k}^{a_{i}}(z) \mathbf{V}_{k}^{a_{i}}=\mathbf{R}_{0 k}^{a_{i}}(z) \mathbf{M}_{k}^{a_{i}}(z)
$$

Сначала докажем аналогичное равенство для операторов $\mathbf{R}_{k j}^{a_{i}}(z)$ и $\mathbf{M}_{k j}^{a_{i}}(z)$. 
Лемма 6. Справедливо равенство

$$
\mathbf{R}_{k j}^{a_{i}}(z) \mathbf{V}_{k}^{a_{i}}=\mathbf{R}_{0 k}^{a_{i}}(z) \mathbf{M}_{k j}^{a_{i}}(z) .
$$

ДокАЗАтЕЛЬство. При $j=N-1$ равенство (48) эквивалентно соотношению

$$
R_{a_{N-1}}(z) V_{a_{N-1}}=R_{0}(z) T_{a_{N-1}}(z)
$$

для парных резольвенты и $T$-матрицы. Для обоснования справедливости перехода $j \rightarrow j-1$ запишем в матричной форме уравнения для операторов $M_{A_{j} B_{j}}^{a_{j-1}}(z)$ :

$$
\mathbf{M}_{k, j-1}^{a_{i}}(z)=\mathbf{M}_{k j}^{a_{i}}(z)-\mathbf{M}_{k j}^{a_{i}}(z) \mathbf{X}_{k j}^{a_{i}} \mathbf{R}_{0 k}^{a_{i}}(z) \mathbf{M}_{k, j-1}^{a_{i}}(z) .
$$

Домножая это равенство слева на $\mathbf{R}_{0 k}^{a_{i}}(z)$ и пользуясь формулой (48), получаем уравнения

$$
\begin{gathered}
\mathbf{R}_{0 k}^{a_{i}}(z) \mathbf{M}_{k, j-1}^{a_{i}}(z)=\mathbf{R}_{k j}^{a_{i}}(z) \mathbf{V}_{k}^{a_{i}}\left(\mathbf{I}_{k}^{a_{i}}-\mathbf{X}_{k j}^{a_{i}} \mathbf{R}_{0 k}^{a_{i}}(z) \mathbf{M}_{k, j-1}^{a_{i}}(z)\right), \\
\left(\mathbf{H}_{k j}^{a_{i}}-z \mathbf{I}_{k}^{a_{i}}\right) \mathbf{R}_{0 k}^{a_{i}}(z) \mathbf{M}_{k, j-1}^{a_{i}}(z)+\mathbf{V}_{k}^{a_{i}} \mathbf{X}_{k j}^{a_{i}} \mathbf{R}_{0 k}^{a_{i}}(z) \mathbf{M}_{k, j-1}^{a_{i}}(z)=\mathbf{V}_{k}^{a_{i}},
\end{gathered}
$$

которые эквивалентны

$$
\mathbf{R}_{k, j-1}^{a_{i}}(z) \mathbf{V}_{k}^{a_{i}}=\mathbf{R}_{0 k}^{a_{i}}(z) \mathbf{M}_{k, j-1}^{a_{i}}(z)
$$

Лемма доказана.

Равенство (47) следует теперь из уравнения (48) при $j=k$ и уравнений (40) и (41). Тем самым соотношение (47) доказано.

Заметим, что формула (48) позволяет переписать уравнение (41) в форме

$$
\mathbf{R}_{k}^{a_{i}}(z)=\mathbf{R}_{0 k}^{a_{i}}(z)-\mathbf{R}_{0 k}^{a_{i}}(z) \mathbf{M}_{k k}^{a_{i}}(z) \mathbf{X}_{k k}^{a_{i}} \mathbf{R}_{k}^{a_{i}}(z)
$$

Как отмечалось выше, уравнения (46) представляют собой дифференциальные уравнения для компонент резольвенты. Запишем их в явном виде:

$$
\begin{aligned}
\left(H_{0}-z\right) R_{A_{k} B_{k}}^{a_{i}}(z) & +V_{a_{N-1}} R_{A_{k} B_{k}}^{a_{i}}(z)+V_{a_{N-1}} \sum_{j=k+1}^{N-1} \sum_{\left(C_{j} \neq A_{j}\right) \subset a_{j-1}}^{\prime} R_{A_{k}^{j-1} C_{j} B_{k}}^{a_{i}}(z)+ \\
& +V_{a_{N-1}} \sum_{d_{k} \neq a_{k}} \sum_{\left(D_{k+1} \neq A_{k+1}\right) \subset a_{k}}^{\prime} R_{D_{k} B_{k}}^{a_{i}}(z)=I \delta_{A_{k} B_{k}}
\end{aligned}
$$

где $H_{0}=-\Delta_{\mathbf{X}}$ и $V_{a_{N-1}}-$ оператор умножения на потенциал $V_{a_{N-1}}\left(\mathbf{x}_{a_{N-1}}\right)$. Уравнения (49) являются обобщением на случай $N$ частиц системы дифференциальных уравнений Фаддеева [27], [28] для $N=3$. Самые сложные уравнения, отвечающие полному гамильтониану $H$ системы $N$ тел, получаются из уравнений (49) при $i=1$ и $k=2$.

Обсудим свойства однородной системы уравнений, отвечающей (49):

$$
\begin{aligned}
\left(H_{0}-z\right) U_{A_{k}}^{a_{i}}+V_{a_{N-1}} U_{A_{k}}^{a_{i}} & +V_{a_{N-1}} \sum_{j=k+1}^{N-1} \sum_{\left(C_{j} \neq A_{j}\right) \subset a_{j-1}}^{\prime} U_{A_{k}^{j-1} C_{j}}^{a_{i}}+ \\
& +V_{a_{N-1}} \sum_{d_{k} \neq a_{k}} \sum_{\left(D_{k+1} \neq A_{k+1}\right) \subset a_{k}}^{\prime} U_{D_{k}}^{a_{i}}=0 .
\end{aligned}
$$


Покажем, что эта система не имеет нетривиальных решений при $\operatorname{Im} z \neq 0$. Перепишем ее в виде

$$
\begin{aligned}
\left(H_{0}-z\right) U_{A_{k}}^{a_{i}} & +V_{a_{N-1}} U_{A_{k}}^{a_{i}}+V_{a_{N-1}} \sum_{j=k+2}^{N-1} \sum_{\left(C_{j} \neq A_{j}\right) \subset a_{j-1}}^{\prime} U_{A_{k}^{j-1} C_{j}}^{a_{i}}+ \\
& +V_{a_{N-1}} \sum_{\left(D_{k+1} \neq A_{k+1}\right) \subset a_{k}}^{\prime} U_{D_{k+1}}^{a_{i}}=0
\end{aligned}
$$

где введено обозначение

$$
\sum_{a_{k} \subset a_{i}} U_{A_{k}}^{a_{i}}=U_{A_{k+1}}^{a_{i}}
$$

Суммируя уравнения (51) по всем $a_{k} \subset a_{i}$, получим более простую систему для компонент $U_{A_{k+1}}^{a_{i}}$, которая получается из (50) заменой $k$ на $k+1$. Продолжая описанную процедуру суммирования, придем к еще более простой системе для компонент $U_{A_{k+2}}^{a_{i}}$ и т. д. На последнем шаге останется лишь одна компонента $U^{a_{i}}=\sum_{A_{k}} U_{A_{k}}^{a_{i}}$, удовлетворяющая уравнению Шредингера

$$
\left(H_{a_{i}}-z\right) U^{a_{i}}=0 .
$$

В силу самосопряженности оператора $H_{a_{i}}$ решение последнего уравнения тривиально при $\operatorname{Im} z \neq 0$, т. е. $U^{a_{i}}=0$.

Покажем теперь, что величины

$$
U_{a_{N-1}}^{a_{i}}=\sum_{A_{k}^{N-2}} U_{A_{k}}^{a_{i}}
$$

равны нулю. Действительно, $U_{a_{N-1}}^{a_{i}}$ удовлетворяют уравнениям

$$
\left(H_{0}-z\right) U_{a_{N-1}}^{a_{i}}=-V_{a_{N-1}} \sum_{b_{N-1}} U_{b_{N-1}}^{a_{i}},
$$

откуда в силу тождества $U^{a_{i}}=\sum_{b_{N-1}} U_{b_{N-1}}^{a_{i}} \equiv 0$ и самосопряженности оператора $H_{0}$ следует, что $U_{a_{N-1}}^{a_{i}}=0$. По этой причине из уравнения (51) при $k=N-2$ мы получаем для компонент $U_{A_{N-2}}^{a_{i}}$ уравнения

$$
\left(H_{a_{i}}+V_{a_{N-1}}-z\right) U_{A_{N-2}}^{a_{i}}=0
$$

и, как и раньше, $U_{A_{N-2}}^{a_{i}}=0$. Продолжая эти рассуждения, заключаем, что все $U_{A_{k}}^{a_{i}}$ равны нулю. Тем самым доказана следующая теорема.

ТЕОрема 2. Спектр операторов $\mathbf{H}_{k}^{a_{i}}$ является вещественным: уравнения

$$
\left(\mathbf{H}_{k}^{a_{i}}-z \mathbf{I}_{k}^{a_{i}}\right) \mathbf{U}_{k}^{a_{i}}=0
$$

при $\operatorname{Im} z \neq 0$ имеют лишь тривиальные решения.

Данная теорема позволяет получить связь между операторами $\mathbf{R}_{k}^{a_{i}}(z)$ и $\mathbf{M}_{k}^{a_{i}}(z)$. Построим оператор $\mathbf{G}_{k}^{a_{i}}(z)$ по формуле

$$
\mathbf{G}_{k}^{a_{i}}(z)=-\mathbf{R}_{0 k}^{a_{i}}(z) \mathbf{M}_{k}^{a_{i}}(z) \mathbf{R}_{0 k}^{a_{i}}(z) .
$$


Из уравнений (40) и (48) получаем следующее уравнение для оператора $\mathbf{G}_{k}^{a_{i}}(z)$ :

$$
\mathbf{G}_{k}^{a_{i}}(z)=-\mathbf{R}_{k k}^{a_{i}}(z) \mathbf{V}_{k}^{a_{i}} \mathbf{R}_{0 k}^{a_{i}}(z)-\mathbf{R}_{k k}^{a_{i}}(z) \mathbf{V}_{k}^{a_{i}} \mathbf{X}_{k}^{a_{i}} \mathbf{G}_{k}^{a_{i}}(z)
$$

или, в “дифференциальной” форме,

$$
\left(\mathbf{H}_{k k}^{a_{i}}-z \mathbf{I}_{k}^{a_{i}}+\mathbf{V}_{k}^{a_{i}} \mathbf{X}_{k k}^{a_{i}}\right) \mathbf{G}_{k}^{a_{i}}(z)=-\mathbf{V}_{k}^{a_{i}} \mathbf{R}_{0 k}^{a_{i}}(z)
$$

Это уравнение для $\mathbf{G}_{k}^{a_{i}}(z)$ и уравнение $(46)$ для $\mathbf{R}_{k}^{a_{i}}(z)$ отличаются лишь свободным членом. Поэтому естественно предположить, что $\mathbf{R}_{k}^{a_{i}}(z)$ выражается линейно через $\mathbf{G}_{k}^{a_{i}}(z)$. Действительно, справедливо такое утверждение.

Лемма 7. Для операторов $\mathbf{R}_{k}^{a_{i}}(z)$ при любом $z, \operatorname{Im} z \neq 0$, верно представление

$$
\mathbf{R}_{k}^{a_{i}}(z)=\mathbf{R}_{0 k}^{a_{i}}(z)+\mathbf{G}_{k}^{a_{i}}(z) \mathbf{Y}_{k}^{a_{i}}
$$

где

$$
\mathbf{Y}_{k}^{a_{i}}=\mathbf{I}_{k}^{a_{i}}+\sum_{m=k}^{N-1} \mathbf{X}_{k m}^{a_{i}}
$$

ДоказАтельство. Рассмотрим оператор $\widehat{\mathbf{R}}_{k}^{a_{i}}(z)=\mathbf{R}_{0 k}^{a_{i}}(z)+\mathbf{G}_{k}^{a_{i}}(z) \mathbf{Y}_{k}^{a_{i}}$. Прямое вычисление приводит к уравнению

$$
\left(\mathbf{H}_{k}^{a_{i}}-z \mathbf{I}_{k}^{a_{i}}\right) \widehat{\mathbf{R}}_{k}^{a_{i}}(z)=\mathbf{I}_{k}^{a_{i}} .
$$

Отсюда, с учетом того что уравнения (52) имеют только тривиальные решения, следует соотношение (54). Лемма доказана.

Запишем формулу (54) в терминах операторов $\mathbf{M}_{k}^{a_{i}}$ :

$$
\mathbf{R}_{k}^{a_{i}}(z)=\mathbf{R}_{0 k}^{a_{i}}(z)-\mathbf{R}_{0 k}^{a_{i}}(z) \mathbf{M}_{k}^{a_{i}}(z) \mathbf{Y}_{k}^{a_{i}} \mathbf{R}_{0 k}^{a_{i}}(z) .
$$

Это равенство является обобщением на случай матричных операторов $\mathbf{H}_{k}^{a_{i}}$ стандартного соотношения $R(z)=R_{0}(z)-R_{0}(z) T(z) R_{0}(z)$ между резольвентой и $T$-матрицей для операторов вида $H=H_{0}+V$. По этой причине оператор

$$
\mathbf{T}_{k}^{a_{i}}(z)=\mathbf{M}_{k}^{a_{i}}(z) \mathbf{Y}_{k}^{a_{i}}
$$

естественно назвать $T$-матрицей для матричного оператора $\mathbf{H}_{k}^{a_{i}}$. Равенства (55) допускают естественное обращение

$$
\mathbf{T}_{k}^{a_{i}}(z)=\mathbf{V}_{k}^{a_{i}} \mathbf{Y}_{k}^{a_{i}}-\mathbf{V}_{k}^{a_{i}} \mathbf{Y}_{k}^{a_{i}} \mathbf{R}_{k}^{a_{i}}(z) \mathbf{V}_{k}^{a_{i}} \mathbf{Y}_{k}^{a_{i}}
$$

и это соотношение вновь является обобщением на матричный случай стандартной формулы $T(z)=V-V R(z) V$.

\section{2. Дифференциальные уравнения для компонент волновых функций.} Операторы $\mathbf{R}_{k}^{a_{i}}(z)$ позволяют построить собственные функции непрерывного спектра операторов $\mathbf{H}_{k}^{a_{i}}$ аналогично тому, как это было сделано в разделе 3. Будем рассматривать полную систему $N$ частиц, которой отвечает случай $i=1, k=2$ и опускать в обозначениях индекс $a_{1}$. Как и в разделе 3 , собственные функции оператора $\mathbf{H}_{2}$ определяются с помощью подходящих пределов функции $\mathbf{R}_{2}(z)$ на 
вещественной оси плоскости переменной $z$. Будем рассматривать пределы функции $\mathbf{R}_{2}(z)$ на верхнем берегу разреза по вещественной оси. Соответствующие функции при этом следует снабдить значком плюс, но мы не будем его указывать, чтобы не перегружать обозначения.

В выбранном нами случае собственные функции непрерывного спектра классифицируются начальными состояниями системы $N$ тел. Вновь будем использовать функции $\chi_{a_{l}}\left(\mathbf{p}_{a_{l}}\right)$, соответствующие $l$ свободным кластерам, $2 \leqslant l<N$, и функцию $\chi_{0}(\mathbf{P})$, соответствующую $N$ свободным частицам (см. раздел 3 ). Введем векторфункции $\boldsymbol{\Psi}^{B_{2}^{l}}\left(\mathbf{p}_{b_{l}}\right)$ с компонентами

$$
\psi_{A_{2}}^{B_{2}^{l}}\left(\mathbf{p}_{b_{l}}\right)=\chi_{A_{l+1}}^{a_{l}}\left(\mathbf{p}_{a_{l}}\right) \delta_{a_{l} b_{l}} \ldots \delta_{a_{2} b_{2}}
$$

и вектор-функции $\boldsymbol{\Psi}_{0}^{B_{2}}(\mathbf{P})$ с компонентами

$$
\psi_{A_{2}}^{B_{2}}(\mathbf{P})=\chi_{0}(\mathbf{P}) \delta_{a_{N-1} b_{N-1}} \ldots \delta_{a_{2} b_{2}} .
$$

Здесь $\chi_{A_{l+1}}^{a_{l}}-$ компоненты функции $\chi_{a_{l}}$, построенные по формулам (16).

Собственные функции непрерывного спектра оператора $\mathbf{H}_{2}$ определяются как пределы

$$
\begin{aligned}
\boldsymbol{\Phi}^{B_{2}^{l}}\left(\mathbf{p}_{b_{l}}\right) & =-\lim _{\epsilon \rightarrow+0} \mathrm{i} \epsilon \mathbf{R}_{2}\left(E_{b_{l}}+\mathrm{i} \epsilon\right) \Psi^{B_{2}^{l}}\left(\mathbf{p}_{b_{l}}\right), \\
\boldsymbol{\Phi}^{B_{2}}(\mathbf{P}) & =-\lim _{\epsilon \rightarrow+0} \mathrm{i} \epsilon \mathbf{R}_{2}\left(E_{0}+i \epsilon\right) \Psi_{0}^{B_{2}}(\mathbf{P}),
\end{aligned}
$$

где $E_{b_{l}}=\mathbf{p}_{b_{l}}^{2}-\varepsilon_{b_{l}}, E_{0}=\mathbf{P}^{2}$. Используя формулу (46) при $k=2, i=1$, получаем из соотношений (57) уравнения

$$
\begin{aligned}
\left(\mathbf{H}_{2}-E_{b_{l}} \mathbf{I}_{2}\right) \boldsymbol{\Phi}^{B_{2}^{l}}\left(\mathbf{p}_{b_{l}}\right) & =0 \\
\left(\mathbf{H}_{2}-E_{0} \mathbf{I}_{2}\right) \boldsymbol{\Phi}^{B_{2}}(\mathbf{P}) & =0
\end{aligned}
$$

которые являются обобщением трехчастичных дифференциальных уравнений Фаддеева [14] на случай системы $N$ частиц. Явная форма уравнений (58) и (59) имеет вид (50) при $z=E_{b_{l}}$ и $z=E_{0}$ соответственно. Собственные функции $\Psi_{b_{l}}^{(+)}$и $\Psi_{0}^{(+)}$ оператора $H$ (см. формулы (24) и (27)) можно восстановить по компонентам функций $\boldsymbol{\Phi}^{B_{2}^{l}}$ и $\boldsymbol{\Phi}^{B_{2}}$ с помощью суммирования:

$$
\Psi_{b_{l}}^{(+)}=\sum_{A_{2}} \varphi_{A_{2}}^{B_{2}^{l}}, \quad \Psi_{0}^{(+)}=\sum_{A_{2}} \varphi_{A_{2}}^{B_{2}} .
$$

Для однозначного определения компонент волновых функций в конфигурационном пространстве уравнения (58) и (59) необходимо дополнить асимптотическими граничными условиями. Эти условия, как и в разделе 4, можно получить с помощью исследования преобразований Фурье соответствующих объектов в импульсном пространстве. Аналогично разделу 4 приведем окончательный результат для компонент функций $\boldsymbol{\Phi}^{B_{2}^{l}}$ при $l=2$. В этом случае цепочка $B_{2}^{l}$ вырождается в одно разбиение $b_{2}$, фиксирующее начальное состояние системы. Асимптотический вид компонент функций $\boldsymbol{\Phi}^{b_{2}}\left(\mathbf{X}, \mathbf{p}_{b_{2}}\right)$ повторяет (38) и дается выражением

$$
\varphi_{A_{2}}^{b_{2}}\left(\mathbf{X}, \mathbf{p}_{b_{2}}\right)=\psi_{A_{3}}^{a_{2}}\left(\mathbf{x}_{a_{2}}\right) e^{\mathrm{i}\left(\mathbf{p}_{a_{2}}, \mathbf{y}_{a_{2}}\right)} \delta_{a_{2} b_{2}}+\sum_{i=2}^{n} \psi_{A_{i+1}}^{a_{i}}\left(\mathbf{x}_{a_{i}}\right) U_{a_{i} b_{2}}^{A^{i-1}}\left(\mathbf{y}_{a_{i}}, \mathbf{p}_{b_{2}}\right)
$$


где $\psi_{A_{i+1}}^{a_{i}}$ - компоненты собственной функции $\psi^{a_{i}}$ оператора $h_{a_{i}}$, построенные по формулам (16). Функции $U_{a_{i} b_{2}}^{A^{i-1}}\left(\mathbf{y}_{a_{i}}, \mathbf{p}_{b_{2}}\right)$ при $\left|\mathbf{y}_{a_{i}}\right| \rightarrow \infty$ переходят в сферические волны:

$$
\begin{aligned}
U_{a_{i} b_{2}}^{A^{i-1}}\left(\mathbf{y}_{a_{i}}, \mathbf{p}_{b_{2}}\right) \sim & C_{3(i-1)}\left(E_{a_{i} b_{2}}\right) \mathcal{H}_{a_{i} b_{2}}^{A^{i-1}}\left(\sqrt{E_{a_{i} b_{2}}} \hat{\mathbf{y}}_{a_{i}}, \mathbf{p}_{b_{2}}, E_{b_{2}}+\mathrm{i} 0\right) \times \\
& \times\left|\mathbf{y}_{a_{i}}\right|^{-(3 i-4) / 2} e^{\mathrm{i} \sqrt{E_{a_{i} b_{2}}}\left|\mathbf{y}_{a_{i}}\right|}
\end{aligned}
$$

где

$$
E_{a_{i} b_{2}}=E_{b_{2}}+\varepsilon_{a_{i}}=\mathbf{p}_{b_{2}}^{2}-\varepsilon_{b_{2}}+\varepsilon_{a_{i}}, \quad C_{n}(E)=-2 \pi^{2} E^{(n-3) / 4} e^{-\mathrm{i} \pi(n-3) / 4} .
$$

Амплитуды $U_{a_{i} b_{2}}^{A^{i-1}}$ и $\mathcal{H}_{a_{i} b_{2}}^{A^{i-1}}$ связаны с сответствующими амплитудами $U_{a_{i} b_{2}}^{+}$и $\mathcal{H}_{a_{i} b_{2}}$ из (38) и (39) формулами

$$
U_{a_{i} b_{2}}^{+}=\sum_{A^{i-1}} U_{a_{i} b_{2}}^{A^{i-1}}, \quad \mathcal{H}_{a_{i} b_{2}}=\sum_{A^{i-1}} \mathcal{H}_{a_{i} b_{2}}^{A^{i-1}} .
$$

\section{6. ЗАКЛЮЧЕНИЕ}

Дано обобщение формализма уравнений Фаддеева в интегральной и дифференциальной формах на случай $N$ частиц. Построены $N$-частичные матричные операторы, обобщающие операторы матричных дифференциальных уравнений Фаддеева для трех частиц. Для этих операторов построены резольвенты и $T$-матрицы, представляющие собой обобщение трехчастичных компонент Фаддеева для резольвенты и $T$-матрицы трехчастичного гамильтониана.

Благодарности. Работа поддержана Программой поддержки ведущих научных школ (грант НШ-1292.2014.1) и РФФИ (грант № 14-02-00326).

\section{Список литературы}

[1] Л. Д. Фаддеев, Математические вопросы квантовой теории рассеяния для системь трех частии, Тр. МИАН СССР, 69, Изд-во АН СССР, М.-Л., 1963.

[2] С. П. Меркурьев, Л. Д. Фаддеев, Квантовая теория рассеяния для систем нескольких частии, Наука, М., 1985.

[3] S. Weinberg, Phys. Rev. B, 133:1 (1964), 232-256.

[4] L. Rosenberg, Phys. Rev. B, 140:1 (1965), 217-226.

[5] A. N. Mitra, J. Gillespie, B. Sugar, N. Panchapakesan, Phys. Rev. B, 140:5 (1965), 1336-1338.

[6] V.A. Alessandrini, J. Math. Phys., 7:2 (1966), 213-220.

[7] J. Weyers, Phys. Rev., 145:4 (1966), 1236-1242.

[8] N. Mishima, J. Takahashi, Prog. Theor. Phys., 35:3 (1966), 440-451.

[9] R. Omnes, Phys. Rev., 165:4 (1968), 1265-1272.

[10] О. А. Якубовский, ЯФ, 5:6 (1967), 1312-1320.

[11] О. А. Якубовский, Тр. МИАН СССР, 110 (1970), 146-177.

[12] K. Hepp, Helv. Phys. Acta, 42 (1969), 425-458.

[13] С. П. Меркурьев, С. Л. Яковлев, Докл. АН СССР, 262:3 (1982), 591-594.

[14] С. П. Меркурьев, С. Л. Яковлев, ТМФ, 56:1 (1983), 60-73.

[15] С. П. Меркурьев, С. Л. Яковлев, ЯФ, 39:6 (1984), 1580-1587.

[16] S. P. Merkuriev, S. L. Yakovlev, C. Gignoux, Nucl. Phys. A, 431:1 (1984), 125-138. 
[17] А. А. Квицинский, Ю. А. Куперин, С. П. Меркурьев, А. К. Мотовилов, С. Л. Яковлев, ЭЧАЯ, 17:2 (1986), 267-317.

[18] С. Л. Яковлев, ТМФ, 82:2 (1990), 224-241.

[19] С. П. Меркурьев, А. К. Мотовилов, С. Л. Яковлев, ТМФ, 94:3 (1993), 435-447.

[20] В. А. Руднев, С. Л. Яковлев, ЯФ, 58:10 (1995), 1762-1771.

[21] С. Л. Яковлев, ТМФ, 102:3 (1995), 323-336.

[22] С. Л. Яковлев, ТМФ, 107:3 (1996), 513-528.

[23] Т. Като, Теория возмущений линейных операторов, Мир, М., 1972.

[24] С. Л. Яковлев, З. Папп, ТМФ, 163:2 (2010), 314-327.

[25] И. М. Народецкий, О. А. Якубовский, "Интегральные уравнения теории рассеяния для $N$ частиц", Проблема нескольких тел в ядерной физике, ОИЯИ, Дубна, 1980, 183-226.

[26] М. Рид, Б. Саймон, Методы современной математической физики, т. 3: Теория рассеяния, Мир, М., 1982.

[27] H. P. Noyes, H. Fiedeldey, "Calculations of three-nucleon low-energy parameters", Three-Particle Scattering in Quantum Mechanics, eds. J. Gillespie, J. Nuttall, W. A. Benjamin, New York, Amsterdam, 1968, 195-293.

[28] S. P. Merkuriev, C. Gignoux, A. Laverne, Ann. Phys., 99:1 (1976), 30-71.

Поступила в редакцию 16.02.2014 